\title{
Berufliche Weiterbildungsbeteiligung von Älteren im europäischen Vergleich - Welche Rolle spielen Institutionen?
}

\author{
Veronika Philipps (D)
}

Eingegangen: 6. Februar 2020 / Angenommen: 24. April 2020 / Online publiziert: 19. Mai 2020

(C) Der/die Autor(en) 2020

Zusammenfassung Personen in der späten Erwerbsphase sind in Europa selten in berufliche Weiterbildung eingebunden. Der Beitrag betrachtet Länderdifferenzen in der Weiterbildungsteilnahme Älterer aus institutionentheoretischer Perspektive und berücksichtigt dabei, dass Institutionen in Verbindung miteinander funktionieren. Daher werden Zusammenhänge zwischen institutionellen Konfigurationen des Arbeitsmarktes und Bildungssystems für die relativen Weiterbildungsnachteile Älterer in 26 europäischen Ländern untersucht und es wird aufgezeigt, dass institutionelle Konfigurationen eine hohe Bedeutung für die Erklärung von Weiterbildungsdisparitäten besitzen. Für geringe Weiterbildungsnachteile Älterer sind insbesondere das Vorhandensein weitreichender staatlicher und betrieblicher Weiterbildungsstrukturen sowie ein duales Berufsausbildungssystem bedeutsam.

Schlüsselwörter Berufliche Weiterbildung · Lebenslanges Lernen ·

Arbeitsmarktinstitutionen · Bildungsinstitutionen · Adult Education Survey ·

Qualitative Comparative Analysis

Dr. V. Philipps $(\bowtie)$

Professur für Wirtschaftspädagogik mit dem Schwerpunkt Berufliches Lehren und Lernen,

Georg-August-Universität Göttingen, Göttingen, Deutschland

E-Mail: veronika.philipps@uni-goettingen.de 


\title{
Job-related further education of the elderly in Europe: Do institutions matter?
}

\begin{abstract}
People in the late employment age are rarely involved in job-related further education in Europe. The article investigates national differences in the participation in continuing education for older people from an institutional-theoretical perspective, taking into account that institutions function in conjunction with one another. Connections between institutional configurations of the labour market and education system for the relative disadvantages of older people in further education in 26 European countries are examined and it is illustrated that institutional configurations are very important for the explanation of disparities in further education. In particular, the existence of far-reaching state and company structures concerning continuing education and a dual vocational training system are crucial for low disadvantages of older people in the adult education system.
\end{abstract}

Keywords Job-related further education · Lifelong Learning · Labour market institutions · Educational institutions · Adult Education Survey · Qualitative Comparative Analysis

\section{Einleitung}

Eine berufliche Weiterentwicklung auch nach dem Abschluss einer Erstausbildung wird als zentraler Bestandteil des Erwerbslebens angesehen und immer wieder von Wissenschaft und Politik auf nationaler und europäischer Ebene betont (z. B. OECD 2019). Insbesondere für Personen in der späten Erwerbsphase ist das Erhalten und Erweitern von beruflichen Fähigkeiten und Wissen wichtig (Becker 2018; Mayer und Solga 2008). Denn Beschäftigte müssen aufgrund des ansteigenden Renteneintrittsalters länger im Erwerbsleben verbleiben und sind somit auf alters- und alternsgerechte Arbeitsbedingungen angewiesen. Zudem können Weiterbildungsmaßnahmen dazu beitragen, vorzeitige Austritte aus dem Erwerbsleben zu reduzieren und somit das Arbeitskräftepotenzial zu erhalten oder auszubauen (Dietz und Walwei 2011; Fourage und Schils 2009; Moraal und Schönfeld 2007). Darüber hinaus gibt es Hinweise, dass ältere Lernende stabilere Beschäftigungsverhältnisse und bessere Chancen auf Wiederbeschäftigung nach einem Arbeitsplatzverlust haben (Bassanini 2006).

Dennoch spiegelt sich die hohe Bedeutung von Weiterbildung für Ältere nicht in der Realität wider: Die Teilnahmequote Älterer (50- bis 60-Jähriger) liegt in Europa mit durchschnittlich $29 \%$ unter derjenigen der 25- bis 49-Jährigen (38\%) (Abb. 1). Gleichzeitig variiert das Ausmaß dieser Altersunterschiede zwischen den Ländern Europas, so dass die Nachteile Älterer gegenüber Jüngeren von Land zu Land unterschiedlich hoch ausfallen: Ältere in der Schweiz weisen z. B. mit einer Teilnahmequote an Weiterbildung von etwa $52 \%$ geringere Nachteile gegenüber Jüngeren $(57 \%)$ auf als es bspw. in Portugal - auf insgesamt niedrigerem Teilnahmeniveau der Fall ist (25\% versus $45 \%)$. 


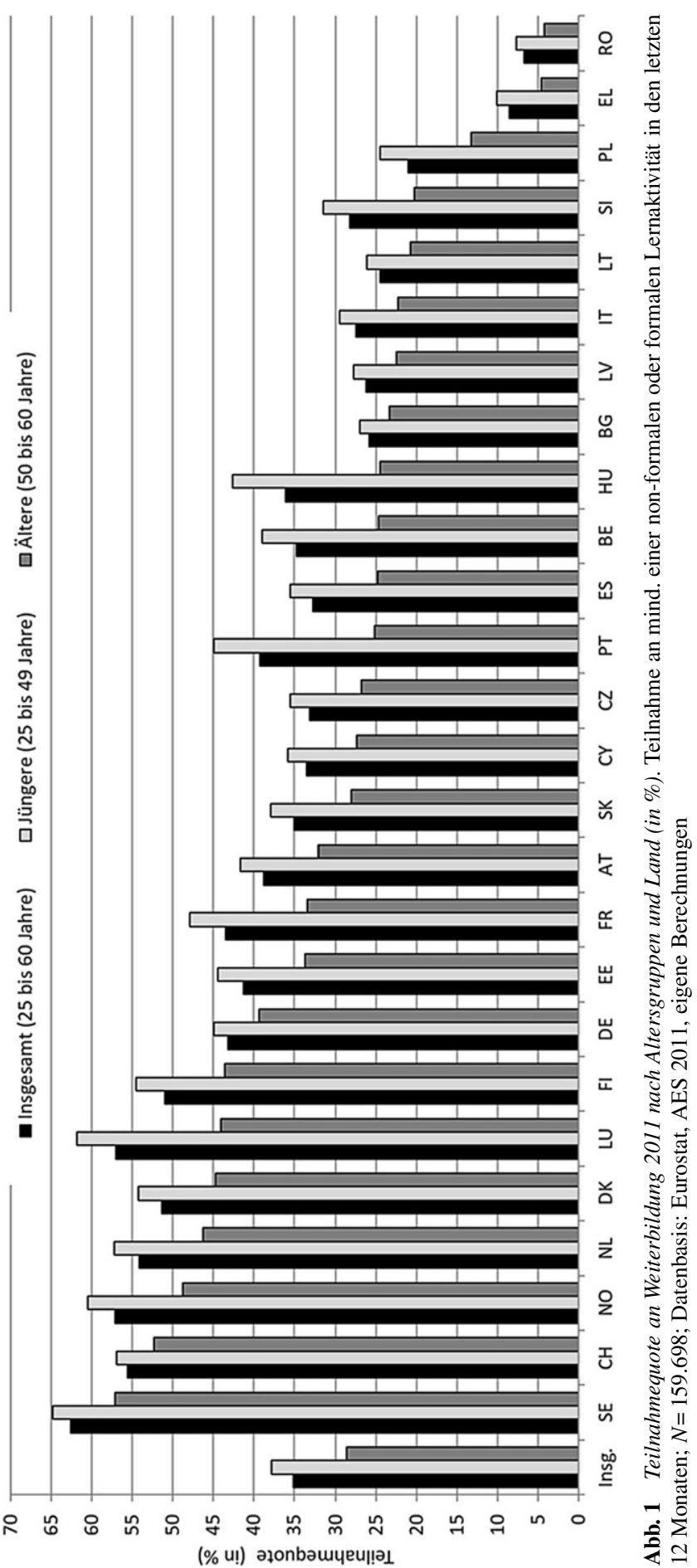


Diese Länderunterschiede werden nicht allein auf individuelle Weiterbildungsentscheidungen zurückzuführen sein, sondern mit der länderspezifischen Ausgestaltung von Institutionen des Arbeitsmarktes und des Bildungssystems zusammenhängen. National unterschiedlich ausgeprägte Institutionen beschränken und ermöglichen soziales Handeln (Scott 2001) und können auch das individuelle und betriebliche Weiterbildungsverhalten beeinflussen. Da Institutionen aufgrund ihrer Komplementarität nicht unabhängig voneinander wirken (Hall und Soskice 2001), geht der Beitrag über die Betrachtung einzelner institutioneller Einflussfaktoren hinaus und nimmt die Rolle von Institutionenkonstellationen für die Weiterbildung Älterer in den Blick. An der unzureichenden Befundlage zu institutionellen Konstellationen und ihrer Wirkung auf die Weiterbildungsteilnahme Älterer setzt der Beitrag an und fragt: Welche Konstellationen institutioneller Bedingungen können die Länderunterschiede in Europa bei den relativen Weiterbildungsnachteilen Älterer erklären?

\section{Weiterbildungsteilnahme Älterer im Ländervergleich: Forschungsüberblick und theoretische Erklärungsansätze}

\subsection{Forschungsstand zu Einflussfaktoren auf die Weiterbildung (Älterer)}

Zahlreiche Studien belegen, dass nicht alle Bevölkerungsgruppen gleichermaßen an Weiterbildung partizipieren. So ist festzuhalten, dass länderübergreifend Personen mit hohem schulischen oder beruflichen Bildungsabschluss eine höhere Weiterbildungsteilnahme aufweisen als jene mit niedrigem Bildungsabschluss (Dämmrich et al. 2014; Martin und Rüber 2016). Die Weiterbildungsbeteiligung ist bei Jüngeren generell höher als bei Älteren (Fourage und Schils 2009), ebenso bei Personen ohne Migrationshintergrund (Kuper et al. 2013; Öztürk 2011). Weniger eindeutig sind die Befunde bezüglich des Geschlechts (Dämmrich et al. 2014; Dieckhoff und Steiber 2011) sowie des Vorhandenseins von Kindern (Dieckhoff und Steiber 2011; Knipprath und De Rick 2015). Das Ausüben einer Erwerbstätigkeit wird dagegen als zentraler Faktor für eine Weiterbildungsteilnahme genannt. So werden in ländervergleichenden Studien tätigkeitsbezogene Merkmale wie eine Vollzeitbeschäftigung (Kaufmann und Widany 2013), eine hohe Zahl an Beschäftigten im Betrieb (Markowitsch und Hefler 2007) sowie die Branche - und hier insbesondere der öffentliche Sektor und einzelne Dienstleistungsbereiche (Arulampalam et al. 2004; Dieckhoff et al. 2007) - als zentrale positive Einflussgrößen auf Weiterbildung identifiziert. In der nachfolgenden Analyse wird daher berücksichtigt, dass sich Länder in ihrer Zusammensetzung nach diesen Einflussfaktoren unterscheiden und solche Kompositionsunterschiede Weiterbildungsdifferenzen zwischen Ländern verursachen können.

Die Rolle institutioneller Rahmenbedingungen für Weiterbildung wurde bisher weniger umfassend untersucht, auch wenn es in der Forschung zu Bildungsaktivitäten Erwachsener zunehmend ländervergleichende Studien gibt (z. B. Boeren und Holford 2016; Ioannidou und Knauber 2019). So wird das Bildungssystem und insbesondere die Ausgestaltung des beruflichen Ausbildungssystems in den Blick genommen. Es gibt Hinweise, dass in stratifizierten Schulsystemen - wie in Deutschland, wo die Bildungswege klar voneinander getrennt sind (Allmendinger 1989; Mül- 
ler und Shavit 1998) und berufliche Spezialisierung in der Erstausbildung stattfindet - die Weiterbildungsteilnahme niedriger ist als in weniger stratifizierten Schulsystemen wie in Großbritannien (Brunello 2004; Groenez et al. 2007; Vogtenhuber 2015). Dagegen sprechen Befunde, die eher ein komplementäres Verhältnis von beruflicher Erstausbildung und Weiterbildung feststellen. So findet Wolbers (2005), dass in Ländern mit einer engen Verbindung von Qualifizierung und Beschäftigung die Weiterbildungsteilnahme höher ist. Ebenso sind Bildungsausgaben des öffentlichen Sektors (jedoch ohne den Weiterbildungsbereich) mit einer höheren Weiterbildungsteilnahme assoziiert (Martin und Rüber 2016). Weitere Studien nehmen den Einfluss der Erwachsenenbildung in den Blick und stellen fest, dass Faktoren wie Zugangsbarrieren, Verfügbarkeit finanzieller Ressourcen, Beratungseinrichtungen und Ausmaß von Angeboten positiv auf die Teilnahme an Weiterbildung wirken (Dämmrich et al. 2014; Groenez et al. 2007).

Zudem werden Institutionen des Arbeitsmarktes und des Wohlfahrtsstaates betrachtet. Es wird konstatiert, dass sich mit steigenden Ausgaben für soziale Sicherung die Weiterbildungsteilnahme in einem Land erhöht (Dämmrich et al. 2014). Auch die Ausrichtung auf eine aktive Arbeitsmarktpolitik bewirkt eine hohe Weiterbildungsbeteiligung (Groenez et al. 2007). Ebenso werden gewerkschaftliche Aktivitäten mit Weiterbildung assoziiert. So findet sich ein positiver Zusammenhang zwischen dem Grad der Gewerkschaftsmitgliedschaft in einem Land und der Weiterbildungsteilnahme (ebd.). Auch bei Beschäftigten in Branchen mit hoher Gewerkschaftsdichte besteht eine höhere Wahrscheinlichkeit der Weiterbildungsteilnahme (Dieckhoff et al. 2007). Dagegen stehen jedoch Befunde, die weniger eindeutig sind und teilweise keine Effekte von Gewerkschaften feststellen (Brunello 2004; Coulombe und Tremblay 2007). Auch der Einfluss des Umfangs des Beschäftigungsschutzes auf Weiterbildung ist nicht ganz eindeutig: Manche Studien konstatieren tendenziell einen negativen Zusammenhang (Bassanini et al. 2005; Brunello 2004), andere Studien bringen gegenteilige Ergebnisse hervor (Almeida und Aterido 2008; Vogtenhuber 2015). Die Ausgestaltung des Rentensystems wird als Institution diskutiert, die speziell auf die Weiterbildung Älterer wirkt. In Ländern mit generösen Frühverrentungsregelungen, die einen frühen Renteneintritt bei hohem Rentenbezug ermöglichen, ist die Weiterbildungsteilnahme Älterer eher gering (Bassanini et al. 2005; Fourage und Schils 2009).

Der Einfluss des wirtschaftlichen Kontextes wird ebenfalls als relevant erachtet. Tendenziell geht eine hohe Arbeitslosenquote mit geringeren Weiterbildungschancen einher (Coulombe und Tremblay 2007; Wolbers 2005). Der Innovationsgrad in einem Land und das Ausmaß wissensintensiver Beschäftigung scheinen dagegen positiv auf die Weiterbildungsbeteiligung zu wirken (Dämmrich et al. 2014; Groenez et al. 2007).

Wenngleich der Forschungsüberblick teilweise widersprüchliche Ergebnisse aufzeigt, sind institutionelle Einflüsse auf die Weiterbildungsbeteiligung deutlich erkennbar. Das wesentliche Forschungsdefizit besteht jedoch darin, dass ausgewählte institutionelle Faktoren lediglich isoliert betrachtet werden, ohne dass institutionelle Komplementaritäten einfließen. Zudem existieren international vergleichende Beiträge vor allem im Bereich der beruflichen Erstausbildung (vgl. Knauber 2017), und Befunde zu strukturellen Bedingungen der Weiterbildung Älterer fehlen weitestge- 
hend. An dieser Forschungslücke setzt der Beitrag an und nimmt die Gruppe der Älteren und ihrer Weiterbildungsbedingungen in den Blick.

\subsection{Institutionelle Erklärungsansätze der Weiterbildungsbeteiligung (Älterer)}

Aus humankapitaltheoretischer Sicht stellt Weiterbildung eine Investition in $\mathrm{Hu}-$ mankapital dar (Becker 1964), die sich für ältere Beschäftigte aufgrund der kürzeren Zeitspanne bis zum Ende des Erwerbslebens nicht mehr lohnen würde (Becker 2018). Diese Argumentation lässt jedoch die Existenz von Länderdifferenzen außer Acht, da die Annahmen der Humankapitaltheorie für alle Länder gleichermaßen Gültigkeit besitzen. Für eine Erklärung der Länderunterschiede in der Weiterbildungsteilnahme (Älterer) bedarf es vielmehr der Integration mehrerer Ebenen: Neben der Mikroebene müssen sowohl die Mesoebene der Weiterbildungsanbieter als auch die Makroebene in den Blick genommen werden (Boeren 2016, S. 146-148). Nach institutionentheoretischen Ansätzen legen Institutionen Grenzen und Optionen für soziales Handeln fest (Scott 2001) und beeinflussen individuelles und betriebliches Handeln (Heinz 1992). Individuen sind sich dabei der Hürden und Gelegenheiten für Lernprozesse bewusst (Cross 1981), nehmen die von den strukturellen Bedingungen hervorgerufenen Opportunitätsstrukturen wahr und interpretieren diese, woraus Entscheidungen für oder gegen eine Weiterbildung resultieren (Evans et al. 2012; Rubenson und Desjardins 2009). Dabei wirken Institutionen aufgrund ihrer Komplementarität nicht unabhängig voneinander. Das Vorhandensein einer Institution kann somit die Funktionalität und Effizienz einer anderen verstärken (Hall und Soskice 2001, S. 17-18).

Institutionen umfassen regulative, normative und kulturell-kognitive Elemente (Scott 2001, S. 48), die auch im Bereich der Weiterbildung existieren. Dies sind Regelungen wie gesetzliche Ansprüche und steuerliche Anreize für Weiterbildungsmaßnahmen, aber auch vorherrschende Vorstellungen und Normen zu Weiterbildung, z. B. ob eine Weiterbildung im späten Erwerbsalter als unangemessen gesehen wird. Ebenso ist die Wahrnehmung und Interpretation von Regelungen und Normen in Form von kulturellen Skripten relevant, wonach Weiterbildungsangebote entsprechend des kulturellen Kontextes interpretiert werden.

Für Weiterbildung zentrale Institutionen sind nicht nur jene des Bildungssystems, sondern auch Institutionen, die den Arbeitsmarkt regulieren und die die Bedingungen der Herausbildung und Nutzung von Qualifikationen beeinflussen (Mayer und Solga 2008). Ein theoretisch relevanter Faktor für die Weiterbildung (Älterer) aus dem Bereich des Bildungssystems sind die länderspezifischen Qualifikationsstrategien verschiedener Ausbildungssysteme, die sich durch eine unterschiedliche Art des Herausbildens von Kompetenzen kennzeichnen (,skill formation“) (Culpepper und Thelen 2008). Diese sind mit der beruflichen Tätigkeit und dem Beschäftigungssystem verbunden. Anzunehmen ist, dass Weiterbildung in Ländern mit einer engen Verbindung von Qualifikation und Beschäftigung und einem standardisierten Berufsbildungssystem, wo die erforderlichen Qualifikationen in der Erstausbildung vermittelt werden, weniger notwendig ist als in Ländern mit einer lockeren Verbindung von Qualifikation und Beschäftigung und einem unstandardisierten Berufsbildungssystem, in dem berufliche Kenntnisse im Betrieb vermittelt und Defizite 
aus der Erstausbildung durch Weiterbildung während des Erwerbslebens kompensiert werden (Brunello 2004; Culpepper und Thelen 2008; Müller und Shavit 1998). Aufgrund der relativ vielen Stellenwechsel zu Beginn des Erwerbslebens kann vermutet werden, dass mehr Einarbeitung in neue Arbeitsplätze nötig ist. In Ländern mit loser Kopplung müsste demnach die Weiterbildungsteilnahme Jüngerer wie Älterer höher sein als in Ländern mit anders strukturierten Bildungssystemen und Arbeitsmärkten. Da insbesondere Jüngere verstärkt an Weiterbildung teilnehmen und damit eine höhere Weiterbildungsteilnahme verzeichnen, müssten die relativen Nachteile für Ältere in der Weiterbildung gering sein.

Einige Argumente beziehen sich auf die Arbeitsmarktstruktur, die für Erstausbildung und Weiterbildung bedeutsam ist (Brunello 2004). Dazu zählt die Ausrichtung der Arbeitsmarktpolitik, die im Falle einer aktiven Ausrichtung Maßnahmen zur Erhaltung von Arbeitsplätzen und Schaffung neuer Arbeitsplätze sowie Weiterbildungsangebote und Arbeitsmarktmaßnahmen - auch für ältere Beschäftigte - fördert (Eurofound 2013). Solche arbeitsmarktpolitischen Maßnahmen zielen vor allem auf die Erhaltung der Erwerbstätigkeit Älterer und die Reduzierung des Anteils an Frühverrentungen (Ebbinghaus und Hofäcker 2013). Da zur Wiedereingliederung in den Arbeitsmarkt und zur Erhaltung der „Beschäftigungsfähigkeit“ Bildungsmaßnahmen besucht werden müssen, wird dies die Weiterbildungsteilnahme erhöhen. Ebenso sind Regelungen zum Übergang in den Ruhestand relevant. Wohlfahrtsstaatliche Regelungen wie Altersrente, Sonderregelungen zur Frühverrentung, Berufsund Erwerbsunfähigkeitsrenten und Arbeitslosengeld beeinflussen das Renteneintrittsverhalten (ebd.). Der Anreiz für Individuen und Betriebe kann bei großzügigen Regelungen den Anreiz zur Weiterbildungsteilnahme bzw. zu Weiterbildungsangeboten verringern, da sich die „Investition“ nicht mehr lohnen würde. Durch die Möglichkeit eines frühen Ausscheidens aus dem Erwerbsleben sollte sich zudem die potenzielle Teilnahme Älterer an Weiterbildung verringern. Ebenso ist anzunehmen, dass weitreichende Regelungen zum Beschäftigungsschutz zu einer erhöhten Arbeitsplatzsicherheit beitragen, da Beschäftigte seltener ausgetauscht werden und somit längere Beschäftigungsverhältnisse bestehen (Acemoglu und Pischke 1999; Estevez-Abe et al. 2001). Argumentiert wird, dass sich Investitionsrisiken für Betriebe verringern und Beschäftigte verstärkt qualifiziert werden, so dass ein strenger Beschäftigungsschutz förderlich für die Weiterbildung Älterer wäre (Dieckhoff 2007). Gewerkschaftliche Aktivitäten können mit Vereinbarungen und Tarifverträgen bezüglich Weiterbildung einhergehen und somit die Gestaltung von Weiterbildungsangeboten in Betrieben regeln (Acemoglu und Pischke 1999; O'Connell und Jungblut 2008). Auch hängen solche Aktivitäten mit der Einbindung von Arbeitnehmervertretungen bezüglich Weiterbildung und mit Weiterbildungsangeboten speziell für benachteiligte Gruppen zusammen (Ok und Tergeist 2003), so dass dies positiv auf die Weiterbildungsteilnahme Älterer wirken sollte.

Generell ist die Weiterbildungsfinanzierung relevant und dabei insbesondere folgende Fragen: Inwieweit sind Maßnahmen staatlich geregelt und finanziert? Oder hängt die Finanzierung vielmehr von den Betrieben oder Individuen selbst ab (Boeren 2016, S. 114-115)? In diesem Zusammenhang spielt die Ausgestaltung des Wohlfahrtsstaates eine Rolle, die das individuelle und betriebliche Investitionsverhalten beeinflussen kann. So lässt sich argumentieren, dass sich Betriebe auf die 
wohlfahrtsstaatliche Absicherung hinsichtlich Arbeitslosenschutz, Lohnabsicherung und Beschäftigungsschutz verlassen; dies hängt mit betrieblichen Qualifizierungsstrategien zusammen (Estevez-Abe et al. 2001). Insofern ist zu erwarten, dass eine höhere wohlfahrtsstaatliche soziale Sicherheit die Bereitschaft, eine Weiterbildung zu verfolgen, erhöht.

Institutionen, die sich speziell auf Ältere beziehen, können insbesondere für die Weiterbildung Älterer theoretisch relevant sein. So ist das Ziel von Arbeitsmarktund Weiterbildungsprogrammen für Ältere, diese Gruppe länger in Erwerbstätigkeit zu halten bzw. wieder ins Erwerbsleben zu integrieren (Eurofound 2013). Ebenso kann das Angebot an umfassenden berufsbezogenen Rehabilitationsmaßnahmen, die auf die Unterstützung des Verbleibs von älteren Beschäftigten im Arbeitsleben zielen sowie die Existenz von Antidiskriminierungsgesetzen bezogen auf die Beschäftigung Älterer, die eine direkte Benachteiligung Älterer verbieten, einen Einfluss haben (ebd.).

Ökonomische Rahmenbedingungen werden als strukturelle Einflussfaktoren von Weiterbildung diskutiert. Ein hohes Ausmaß an wissensintensiver Beschäftigung und innovativen Branchen kann einen höheren Bedarf an Qualifizierung und Weiterbildung bewirken (Vogtenhuber 2015). Wenn das Angebot an qualifizierten Fachkräften gering ist, kann dies die Weiterbildungsbereitschaft in Betrieben erhöhen, um ihren Bedarf an Fachkräften zu decken. Eine schlechte Wirtschaftslage kann die Bereitschaft zu Weiterbildungsinvestitionen einschränken (Markowitsch und Hefler 2007).

Die institutionellen Faktoren lassen sich zu theoretischen Konstrukten - im Folgenden „Faktorenbündel“ - zusammenfassen, da in der geplanten Analyse nur eine begrenzte Anzahl an Bedingungen aufgenommen werden kann (Schneider und

Tab. 1 Institutionelle Einflussfaktoren der Weiterbildungsteilnahme und institutionelle Faktorenbündel. Eigene Darstellung

\begin{tabular}{|c|c|c|}
\hline Institutioneller Einflussfaktor & Faktorenbündel & $\begin{array}{l}\text { Faktorenbündel in der } \\
\text { Analyse }\end{array}$ \\
\hline $\begin{array}{l}\text { Ausrichtung der Arbeitsmarktpolitik } \\
\text { Beschäftigungsschutzmaßnahmen } \\
\text { Ruhestandsregelungen und Frühverrentungs- } \\
\text { möglichkeiten }\end{array}$ & Arbeitsmarktpolitik & $\begin{array}{l}\text { Beschäftigungsfördernde } \\
\text { Arbeitsmarktpolitik } \\
(A M P)\end{array}$ \\
\hline $\begin{array}{l}\text { Berufliches Bildungssystem/berufliche Qualifi- } \\
\text { zierungsstrategien }\end{array}$ & $\begin{array}{l}\text { Qualifikation und Be- } \\
\text { schäftigungssystem }\end{array}$ & $\begin{array}{l}\text { Lockere Verbindung } \\
\text { von Qualifikation und } \\
\text { Beschäftigungssystem } \\
(Q U A L)\end{array}$ \\
\hline $\begin{array}{l}\text { Staatliche Finanzierung von Weiterbildung } \\
\text { Betriebliche Finanzierung von Weiterbildung } \\
\text { Gewerkschaftliche Bestimmungen bzgl. Wei- } \\
\text { terbildung }\end{array}$ & $\begin{array}{l}\text { Staatliche und betrieb- } \\
\text { liche Weiterbildungs- } \\
\text { struktur }\end{array}$ & $\begin{array}{l}\text { Gut ausgebaute staat- } \\
\text { liche und betriebliche } \\
\text { Weiterbildungsstruktur } \\
(W B S T R)\end{array}$ \\
\hline $\begin{array}{l}\text { Arbeitsmarkt- und Weiterbildungsprogramme } \\
\text { für Ältere } \\
\text { Berufsbezogene Rehabilitationsmaßnahmen } \\
\text { Antidiskriminierungsgesetze bzgl. Beschäfti- } \\
\text { gung Älterer }\end{array}$ & $\begin{array}{l}\text { Inkludierende/ } \\
\text { exkludierende staat- } \\
\text { liche Regulierungen } \\
\text { bezüglich Älterer }\end{array}$ & $\begin{array}{l}\text { Inkludierende staatliche } \\
\text { Regulierungen bezüglich } \\
\text { Älterer }(I N K L)\end{array}$ \\
\hline $\begin{array}{l}\text { Wirtschaftliche Lage } \\
\text { Wissensintensive Beschäftigung } \\
\text { Angebot an qualifizierten Arbeitskräften }\end{array}$ & $\begin{array}{l}\text { Ökonomische Rahmen- } \\
\text { bedingungen }\end{array}$ & - \\
\hline
\end{tabular}


Wagemann 2007, S. 101-104). Die als theoretisch relevant identifizierten institutionellen Faktoren werden also durch Faktorenbündel repräsentiert und für die Analyse entsprechend der Annahme benannt, in welche Richtung sie als Bedingungen auf geringe relative Nachteile Älterer in der Weiterbildung wirken (Tab. 1). Das Faktorenbündel „ökonomische Rahmenbedingungen“ wird nicht separat analysiert, da es bereits durch Indikatoren der anderen Faktorenbündel mit abgebildet wird (Abschn. 3.2.2).

\subsection{Theoretische Erwartungen}

Die vier institutionellen Faktorenbündel werden als notwendige und/oder hinreichende Bedingungen für geringe relative Nachteile Älterer in der Weiterbildung (WBREL) untersucht. Eine Bedingung ist dann notwendig, wenn sie immer auch vorliegt, wenn WBREL auftritt. Sie ist also die Voraussetzung für das Eintreten geringer relativer Weiterbildungsnachteile Älterer. Eine Bedingung ist hinreichend, wenn bei deren Erfüllung zwangsweise gefolgert werden kann, dass WBREL auch eintritt, d.h. die vorliegende Bedingung führt auch immer zu WBREL (Abschn. 3.2.1).

Generell ist anzunehmen, dass eine beschäftigungsfördernde Arbeitsmarktpolitik $(A M P)$, eine lockere Verbindung von Qualifikation und Beschäftigungssystem $(Q U A L)$, eine gut ausgebaute staatliche und betriebliche Weiterbildungsstruktur (WBSTR) und inkludierende staatliche Regulierungen bezüglich Älterer (INKL) geringe relative Weiterbildungsnachteile Älterer hervorrufen.

H1: Eine beschäftigungsfördernde Arbeitsmarktpolitik ist eine Voraussetzung für geringe relative Weiterbildungsnachteile Älterer, da Ältere durch einen hohen Beschäftigungsschutz, Ruhestandsregelungen für einen späteren Renteneintritt und durch aktive arbeitsmarktpolitische Maßnahmen länger im Erwerbsleben bleiben und dadurch Zugangsmöglichkeiten zu Weiterbildung erhalten. Daher wird AMP als Voraussetzung und damit als notwendig für geringe relative Weiterbildungsnachteile Älterer angesehen.

H2: Eine lockere Verbindung von Qualifikation und Beschäftigungssystem bewirkt eine höhere Weiterbildungsteilnahme Älterer, da Defizite aus der Erstausbildung durch Weiterbildung ausgeglichen werden, so dass Weiterbildung kontinuierlich im Erwerbsleben - insbesondere für Jüngere aufgrund häufiger Stellenwechsel und Einarbeitung in der frühen Erwerbsphase - stattfindet. Demnach ist anzunehmen, dass sich $Q U A L$ als hinreichend für geringe Nachteile Älterer in der Weiterbildung zeigt.

H3: Gut ausgebaute staatliche und betriebliche Weiterbildungsstrukturen spielen eine wesentliche Rolle für die Weiterbildungsteilnahme und ermöglichen zudem auch Älteren, an Weiterbildung zu partizipieren. Daher wird sich WBSTR als hinreichende Bedingung für geringe Weiterbildungsnachteile Älterer gegenüber Jüngeren zeigen. 
H4: Inkludierende staatliche Regulierungen spielen eine besondere Rolle für die Weiterbildungsteilnahme Älterer, da in diesem Fall die Gruppe der Älteren in besonderer Weise berücksichtigt und speziell gefördert sowie eine Benachteiligung Älterer gesetzlich (und kulturell) missbilligt bzw. geahndet wird. Daher sollte INKL als hinreichende Bedingung für geringe relative Weiterbildungsnachteile Älterer auftreten.

H5: Aufgrund der institutionellen Komplementarität wird erwartet, dass das Auftreten mehrerer dieser Faktorenbündel in Kombination die Weiterbildungsteilnahme Älterer begünstigt. Daher sollten die Faktorenbündel in Kombination als hinreichende Bedingungen auftreten.

\section{Methodisches Vorgehen und Daten}

\subsection{Erster Analyseschritt}

Um die Forschungsfrage beantworten zu können, werden zunächst die Nachteile Älterer gegenüber Jüngeren in der Weiterbildung ermittelt. Dafür werden Kompositionseffekte auf die Weiterbildungsteilnahme berücksichtigt. Denn Merkmale wie der erreichte Bildungsabschluss, der einen Einfluss auf das individuelle Weiterbildungsverhalten hat, ist zwischen den Ländern unterschiedlich ausgeprägt und kann Länderdifferenzen verursachen. Als Datengrundlage dient der Adult Education Survey (AES) von Eurostat, der zwischen Juli 2011 und Juni 2012 individuelle Weiterbildungsaktivitäten von Personen zwischen 25 und unter 65 Jahren in 30 europäischen Staaten erhebt. ${ }^{1}$ Aufgrund der teilweise abweichenden Datenerhebung müssen das Vereinigte Königreich und Irland aus der Analyse ausgeschlossen werden. Zudem fehlen Makrodaten zur institutionellen Ausgestaltung von Malta und Serbien, so dass hier 26 Länder $(N=159.698)$ ausgewertet werden.

Zur Berechnung der relativen Nachteile Älterer unter Berücksichtigung von Kompositionsunterschieden werden binär logistische Regressionen je Land unter Kontrolle von folgenden individuellen und tätigkeitsbezogenen Merkmalen durchgeführt: höchster erreichter allgemeinbildender und beruflicher Abschluss, Migrationshintergrund, Geschlecht, Anzahl jüngerer Kinder im Haushalt, Erwerbstätigkeit nach den Beschäftigungsmerkmalen Betriebsgröße und Branche. Die abhängige Variable wird als Teilnahmequote an Weiterbildung operationalisiert. Als Teilnehmende zählen 25- bis 60-Jährige, wenn sie in den letzten 12 Monaten an mindestens einer non-formalen und/oder formalen Lernaktivität zu beruflichen Zwecken teilgenommen haben. ${ }^{2}$ Darunter fallen sowohl der Besuch von Bildungsgängen als auch Kurse außerhalb des formalen Bildungssystems, die nicht unbedingt $\mathrm{zu}$ einem formalen

\footnotetext{
1 Der AES 2011 umfasst alle 27 EU-Mitgliedsstaaten sowie Norwegen, die Schweiz und Serbien. Zukünftig wird der AES alle fünf Jahre durchgeführt (European Commission/Eurostat 2013, S. 8). Das Erkenntnisinteresse besteht hier darin, die Weiterbildung durch institutionelle Bedingungskonstellationen zu erklären, wofür aktuellere Daten nicht notwendig sind.

2 Für die gemeinsame Betrachtung von non-formalem und formalem Lernen spricht, dass es im Ländervergleich ein unterschiedliches Verständnis von Weiterbildung gibt und daraus teilweise abweichende Zuordnungsweisen zu den Lernformen resultieren (Behringer und Schönfeld 2014, S. 385).
} 
Abschluss führen. Um den Effekt des Alters zu berechnen, wird als unabhängige Variable das Alter mit den Ausprägungen 25- bis 49-Jährige (,Jüngere“) und 50- bis 60-Jährige (,Ältere“) genutzt. Damit wird die Lebensphase umfasst, in der Ältere in der Regel noch erwerbstätig und nicht im Ruhestand sind bzw. nicht unmittelbar vor ihrem Renteneintritt stehen. Aus den logistischen Regressionen je Land ergeben sich vorhergesagte Wahrscheinlichkeiten, auf deren Grundlage der Quotient der geschätzten Teilnahmequote Älterer und der geschätzten Teilnahmequote Jüngerer berechnet wird. ${ }^{3}$ Diese relative Teilnahmewahrscheinlichkeit beschreibt das Ausmaß der relativen Weiterbildungsnachteile Älterer je Land (unabhängig von Kompositionsunterschieden) und wird im zweiten Analyseschritt weiter genutzt.

\subsection{Zweiter Analyseschritt}

\subsubsection{Methodisches Vorgehen}

Im zweiten Analyseschritt werden die institutionellen Einflüsse auf der Länderebene fokussiert und untersucht, welche institutionellen Konstellationen die Länderunterschiede bei den relativen Nachteilen Älterer in der Weiterbildung bedingen. Mit dem hier angewandten Verfahren der Qualitative Comparative Analysis (QCA) kann auf Basis mengentheoretischer Beziehungen die Wirkung von (Kombinationen von) Bedingungen auf ein zu erklärendes Phänomen (Outcome) erforscht werden (Ragin 1987, 2009). Dazu wird untersucht, welche Kombinationen von Institutionen (Bedingungen) als notwendig und/oder hinreichend für geringe relative Nachteile Älterer in den europäischen Ländern (Fälle) identifiziert werden. Eine Bedingung ist dann notwendig, wenn sie immer auch vorliegt, wenn geringe relative Weiterbildungsnachteile Älterer (Outcome) auftreten. Tritt also das Outcome ein, ist notwendigerweise auch die Bedingung erfüllt. Die Bedingung ist in diesem Fall also die Voraussetzung für das Eintreten eines Outcomes. Eine Bedingung ist hinreichend, wenn bei deren Erfüllung gefolgert werden kann, dass das Outcome auch eintritt, d.h. die vorliegende Bedingung führt auch immer zum Outcome (Schneider und Wagemann 2007).

Im Gegensatz zu anderen quantitativen Verfahren lässt sich mit der QCA nicht nur der Einfluss isolierter Faktoren betrachten, sondern die Wirkung von Kombinationen von Institutionen. Eine QCA berücksichtigt, dass die Wirkung einer Bedingung erst mit weiteren Faktoren eintreten kann und dass verschiedene Bedingungen zum selben Outcome führen können (,Äquifinalität“) (Wagemann und Schneider 2010, S. 385-387). Basierend auf Bool'scher Algebra und mengentheoretischen Beziehungen zwischen Outcome und Bedingungen - im Vergleich zu quantitativen Verfahren mit linearer Algebra und additiven Beziehungen -, stellt sie eine explorative Methode dar, die als Ausgangspunkt für weitere Forschung dienen kann. ${ }^{4}$

\footnotetext{
${ }^{3}$ Eine relative Teilnahmewahrscheinlichkeit von 1 würde eine identische Teilnahmequote beider Altersgruppen bedeuten, Werte unter 1 drücken einen Nachteil Älterer gegenüber Jüngeren aus. Damit wird die Wahrscheinlichkeit der Älteren sowie der Jüngeren geschätzt, wenn alle anderen Variablen im Modell auf ihrem jeweiligen Mittelwert gehalten werden.

${ }^{4}$ QCA ist bereits für die Analyse von Bildungssystemen angewendet worden (z. B. Borgna 2017). Für einen Überblick über Anwendungsbereiche in der Soziologie siehe Buche und Siewert (2015).
} 
Um Zusammenhänge zwischen Kombinationen von Bedingungen und dem Outcome aufdecken zu können, wird den Fällen (hier Länder) der Grad ihrer Zugehörigkeit zu einer Menge im Outcome und in den Bedingungen (als „Mitgliedschaft“ bezeichnet) zugewiesen. Dies geschieht mithilfe der sogenannten Kalibrierung, die der Operationalisierung bei anderen Verfahren ähnelt. Dazu werden die Werte der Ausgangsvariablen in „Fuzzy-Werte“ zwischen 0 für keine Mitgliedschaft und 1 für volle Mitgliedschaft in der Menge transformiert. Anschließend wird geprüft, welche Bedingungskombinationen in der Realität auftauchen (dargestellt in der ,Wahrheitstabelle") und es lassen sich die empirisch vorhandenen Konfigurationen auf ihre kürzeste Lösung reduzieren. ${ }^{5}$ Diese beschreibt die identifizierten notwendigen und/oder hinreichenden Bedingungen und lässt sich anhand folgender Gütemaße, die zwischen 0 und 1 variieren, beurteilen (Schneider und Wagemann 2007, S. 203-211): Die Konsistenz gibt an, inwieweit eine Konfiguration hinreichend für das Auftreten des Outcomes ist (Anpassungsgüte; beim Wert 1 wäre die Teilmengenbeziehung perfekt geschätzt). Die Abdeckung zeigt, welcher Anteil des zu erklärenden Outcomes von der hinreichenden Bedingung erklärt wird.

\subsubsection{Daten und Kalibrierung}

Das Outcome „Geringe relative Nachteile Älterer in der Weiterbildung“ (kurz WBREL) wird auf Basis der um Kompositionsunterschiede bereinigten relativen Weiterbildungsnachteile Älterer aus dem ersten Analyseschritt (Abschn. 3.1) kalibriert. Die Ausgangswerte für relative Nachteile werden anhand von drei Ankerpunkten in Fuzzy-Werte überführt: Erstens wird die Schwelle zur vollen Mitgliedschaft in der Menge WBREL zwischen Dänemark und Norwegen bei 0,81 gesetzt (Abb. 2). Zweitens wird am unteren Ende der Bruch in der Verteilung zwischen Portugal und Ungarn $(0,48)$ als Ankerpunkt für eine Nicht-Mitgliedschaft genutzt. Damit werden alle Länder, in denen die Wahrscheinlichkeit einer Weiterbildungsteilnahme Älterer weniger als die Hälfte der Jüngeren beträgt, als kein Mitglied der Länder mit geringen Weiterbildungsnachteilen Älterer definiert. Drittens wird als Indifferenzpunkt der Durchschnitt von 0,63 gesetzt, der den Punkt mit der größten Ambiguität wiedergibt. Werte zwischen den Ankerpunkten werden mithilfe einer logistischen Funktion zugewiesen. ${ }^{6}$ Tab. 2 gibt einen Überblick über die Ankerpunkte von Outcome und Bedingungen.

Die verwendeten Indikatoren sowie die Kalibrierung der Bedingungen werden aus Platzgründen hier nicht ausführlich dargestellt und können im Detail bei Philipps (2019) nachvollzogen werden (einen Überblick über Indikatoren, Datenbasis und Ankerpunkte bieten Abb. 5, 6, 7 und 8 im Anhang). Eine beschäftigungsfördernde Arbeitsmarktpolitik $(A M P)$ wird anhand von zwei Indikatoren kalibriert: Erstens ,aktive Arbeitsmarktpolitik“ ( $A K T)$, gemessen an den Ausgaben für aktive arbeitsmarktpolitische Maßnahmen (in \% des BIP) je 1\% Arbeitslosigkeit und zweitens

\footnotetext{
5 Dies wird mithilfe des Quine McCluskey-Algorithmus durchgeführt. Die Analyse erfolgt mit dem Programm $f_{s} Q C A 3.0$ (Ragin und Davey 2016).

${ }^{6}$ Robustheitstests beim Festlegen der Ankerpunkte bringen weitestgehend stabile Ergebnisse hervor (vgl. Philipps 2019, Anhang D).
} 


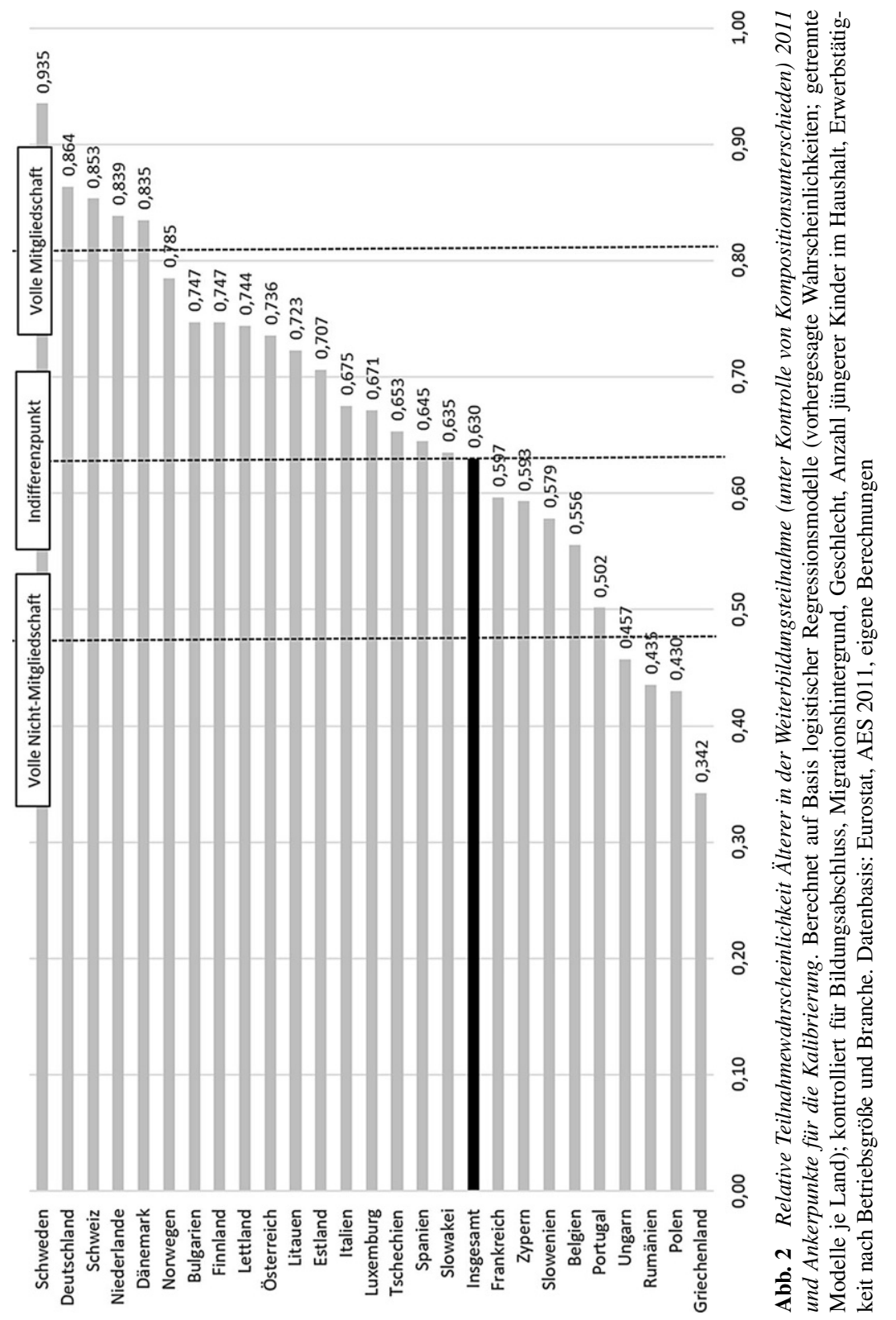


Tab. 2 Messung und Kalibrierung von Outcome und institutionellen Bedingungen. Eigene Darstellung

\begin{tabular}{|c|c|c|c|c|}
\hline \multirow[t]{2}{*}{ Menge } & \multirow[t]{2}{*}{ Messung } & \multicolumn{3}{|c|}{$\begin{array}{l}\text { Kalibrierung (Mitgliedschaft in der } \\
\text { Menge) }\end{array}$} \\
\hline & & $\begin{array}{l}\text { Nicht- } \\
\text { Mitglied- } \\
\text { schaft }\end{array}$ & $\begin{array}{l}\text { Indif- } \\
\text { ferenz- } \\
\text { punkt }\end{array}$ & $\begin{array}{l}\text { Volle } \\
\text { Mitglied- } \\
\text { schaft }\end{array}$ \\
\hline $\begin{array}{l}\text { WBREL: Geringe } \\
\text { relative Nachteile } \\
\text { Älterer in der WB }\end{array}$ & $\begin{array}{l}\text { Relative Nachteile Älterer (Quotient } \\
\text { geschätzte Teilnahmequote Älterer } \\
\text { und Jüngerer) }\end{array}$ & 0,48 & 0,63 & 0,81 \\
\hline \multirow[t]{2}{*}{$\begin{array}{l}A M P: \text { Beschäfti- } \\
\text { gungsfördernde } \\
\text { Arbeitsmarktpolitik }\end{array}$} & $\begin{array}{l}\text { Ausgaben aktive AMP (in } \% \text { des } \\
\text { BIP) je } 1 \% \text { Arbeitslosigkeit }(A K T) \\
\text { UND }\end{array}$ & 0,035 & 0,08 & 0,208 \\
\hline & $\begin{array}{l}\text { Beschäftigungsschutzindex } \\
(B E S C H)\end{array}$ & 1,60 & 2,31 & 2,92 \\
\hline $\begin{array}{l}\text { QUAL: Lockere Ver- } \\
\text { bindung Qualifikation } \\
\text { und Beschäftigung }\end{array}$ & $\begin{array}{l}\text { Anteil Schüler/innen in Sek. II in } \\
\text { kombiniertem schulischen und } \\
\text { betrieblichen Bildungsgang (in \%) }\end{array}$ & 39,95 & 13,95 & 0,15 \\
\hline \multirow[t]{2}{*}{$\begin{array}{l}\text { WBSTR: Gute } \\
\text { staatliche und } \\
\text { betriebliche Weiterbil- } \\
\text { dungsstruktur }\end{array}$} & $\begin{array}{l}\text { Betriebliche Ausgaben für Weiter- } \\
\text { bildung je Beschäftigtem } \\
\text { (WBBETR) } \\
\text { UND }\end{array}$ & 61,5 & 223,0 & 371,5 \\
\hline & $\begin{array}{l}\text { Umfang staatlicher Angebote an } \\
\text { Weiterbildung (WBSTAAT) }\end{array}$ & $\begin{array}{l}\text { Sehr } \\
\text { gering }\end{array}$ & - & $\begin{array}{l}\text { Sehr } \\
\text { hoch }\end{array}$ \\
\hline $\begin{array}{l}\text { INKL: Inkludierende } \\
\text { staatliche Regulierun- } \\
\text { gen für Ältere }\end{array}$ & $\begin{array}{l}\text { Umfang Arbeitsmarkt- und Weiter- } \\
\text { bildungsprogramme für Ältere }\end{array}$ & $\begin{array}{l}\text { Sehr } \\
\text { gering }\end{array}$ & - & $\begin{array}{l}\text { Sehr } \\
\text { hoch }\end{array}$ \\
\hline
\end{tabular}

Beschäftigungsschutz, abgebildet anhand des Beschäftigungsschutzindexes bezüglich individueller Entlassungen im Rahmen unbefristeter Beschäftigung (BESCH). Beide Indikatoren werden als konstitutive Bestandteile einer beschäftigungsfördernden Arbeitsmarktpolitik gesehen und im Faktorenbündel AMP kombiniert. ${ }^{7}$ Auf den Indikator „Alter des erstmaligen Rentenbezugs“ wird in diesem Faktorenbündel aufgrund des hohen Zusammenhangs mit aktiver Arbeitsmarktpolitik und seiner geringeren Varianz verzichtet (Philipps 2019, S. 104-105).

Die Bedingung „Lockere Verbindung von Qualifikation und Beschäftigungssystem" (QUAL) wird basierend auf dem Anteil der Schülerinnen und Schüler in beruflichen Ausbildungsgängen im Sekundarbereich II, bei denen in der Schule und im Betrieb vermittelte Elemente kombiniert werden, kalibriert. Ein niedriger Anteil kennzeichnet eine lockere Verbindung von Qualifikation und Beschäftigung, ein hoher Anteil eine enge Verbindung bzw. ein duales Ausbildungssystem. Die berufliche Ausbildung auf mittlerer Qualifikationsebene stellt hier also den zentralen Faktor dar und repräsentiert das Faktorenbündel, wenngleich andere Merkmale der Erstausbildung wie die schulische Berufsausbildung nicht mit einfließen.

Für die Bedingung „Gut ausgebaute staatliche und betriebliche Weiterbildungsstruktur“ (WBSTR) ist entscheidend, dass sowohl die betriebliche Finanzierung von

\footnotetext{
7 Dies geschieht durch eine Intersektion, die auf Basis des logischen UND berechnet wird (Schneider und Wagemann 2007, S. 185-189). Dafür wird das Minimum der Fuzzy-Werte der Einzelfaktoren herangezogen (s. Tab. 4 im Anhang).
} 
Weiterbildung (WBBETR) als auch der Umfang staatlicher Angebote an Weiterbildung (WBSTAAT) ausgeprägt sind, so dass diese im Faktorenbündel WBSTR kombiniert werden. Betriebliche Weiterbildungsausgaben lassen sich anhand der direkten Kosten der Unternehmen für Weiterbildung je Beschäftigtem abbilden, für den Umfang staatlicher Angebote an Weiterbildung fehlen dagegen geeignete Indikatoren. Daher werden qualitative Länderstudien ausgewertet, die Informationen zum Ausmaß von Rechtsansprüchen und Instrumenten wie individuelle Lernkonten, Bildungsurlaube, Weiterbildungsfonds und steuerliche Anreize für Individuen und Betriebe einbeziehen. ${ }^{8}$ Basierend auf diesem Wissen über die Länder wird nach der ,indirekten Methode“ (Ragin 2009) in der Form kalibriert, dass die Länder Fuzzy-Werten auf einer sechsstufigen Skala von „sehr geringem“ bis „sehr hohem Umfang staatlicher Weiterbildungsangebote" zugeordnet werden. Auch wenn dafür teilweise verschiedene Datenquellen herangezogen wurden, so kann basierend auf den erstellten Länderportraits (Philipps 2019, S. 108-129) das Ausmaß staatlicher Weiterbildungsangebote untersucht werden.

Für „Inkludierende staatliche Regulierungen bezüglich der Weiterbildung Älterer“ (INKL) werden qualitative Informationen aus einer Studie zu Arbeitsmarkt- und Weiterbildungsprogrammen für Ältere (Eurofound 2013) herangezogen und untersucht, inwieweit Weiterbildungsprogramme speziell für Ältere und für arbeitslose Ältere, Beschäftigungsprogramme in Form von subventionierten Arbeitsplätzen für ältere Beschäftigte, Regelungen bezüglich der Kostenübernahme oder Steuerermäßigungen für Betriebe bei Einstellung Älterer sowie Subventionen für die Beschäftigung älterer Arbeitsloser existieren. Auch wenn damit das Problem des Fehlens quantifizierbarer Informationen umgangen werden kann, wären weitere Informationen zur Einordnung und Wirkung der Programme nützlich gewesen. Daher lässt sich INKL nur auf einer weniger kleinteiligen vierstufigen Fuzzy-Skala von „sehr geringem“ bis „,sehr hohem Umfang“ kalibrieren.

Das Faktorenbündel „ökonomische Rahmenbedingungen“ wird bereits durch Indikatoren anderer Faktorenbündel abgebildet, so dass dieses nicht separat analysiert wird. So weisen das BIP pro Kopf sowie der Beschäftigtenanteil in wissensintensiven Branchen in einem Land einen hohen Zusammenhang mit den Ausgaben für aktive Arbeitsmarktpolitik auf (Philipps 2019, S. 138-142). Zudem fehlen geeignete Daten oder qualitative Informationen für gewerkschaftliche Bestimmungen bezogen auf Weiterbildung sowie für berufliche Rehabilitationsmaßnahmen und Antidiskriminierungsgesetze bezüglich der Beschäftigung Älterer (ebd.).

Um das generelle Weiterbildungsniveau eines Landes bei der Analyse der Bedingungen zu berücksichtigen, wird das Untersuchungssample - anhand der durchschnittlichen Weiterbildungsquote der Bevölkerung - in 13 Länder mit hohem und 13 Länder mit niedrigem Weiterbildungsniveau aufgeteilt. ${ }^{9}$

\footnotetext{
8 Als Hauptquelle dient die Datenbank vom European Centre for the Development of Vocational Training (CEDEFOP) „Financing adult learning“ (http://www.cedefop.europa.eu/FinancingAdultLearning, letzter Zugriff 27.01.2020), sowie die Länderberichte „VET in Europe“ von CEDEFOP.

${ }^{9}$ Länder mit hohem Weiterbildungsniveau: Dänemark, Deutschland, Estland, Finnland, Frankreich, Luxemburg, Niederlande, Norwegen, Österreich, Portugal, Schweden, Schweiz, Ungarn; Länder mit niedrigem Weiterbildungsniveau: Belgien, Bulgarien, Griechenland, Italien, Lettland, Litauen, Polen, Rumänien, Slowakei, Slowenien, Spanien, Tschechien, Zypern.
} 


\section{Institutioneller Erklärungsbeitrag der Weiterbildungsnachteile Älterer}

Wie hoch sind die Weiterbildungsnachteile Älterer gegenüber Jüngeren - bereinigt um die Kompositionseffekte - in den europäischen Ländern? Ältere haben im Vergleich zu Jüngeren im Durchschnitt aller untersuchten Länder eine geringere Teilnahmewahrscheinlichkeit von 0,63 (Abb. 3). Damit beträgt die Wahrscheinlichkeit für Ältere, an einer Weiterbildung teilzunehmen, weniger als zwei Drittel im Vergleich zu Jüngeren. Das Ausmaß dieser relativen Nachteile findet auf ganz unterschiedlichen Weiterbildungsniveaus statt und variiert zwischen Schweden am oberen Ende mit der höchsten relativen Teilnahmewahrscheinlichkeit Älterer $(0,94)$ - und damit den geringsten Nachteilen - und am unteren Ende Griechenland mit der geringsten relativen Teilnahmewahrscheinlichkeit Älterer in der Weiterbildung $(0,34)$. Demnach lassen sich die Länderdifferenzen nur teilweise auf Kompositionseffekte zurückführen.

Für die Analyse der institutionellen Konfigurationen und ihrer Rolle für die zuvor bestimmten Weiterbildungsnachteile Älterer werden nun für die beiden Untersuchungssamples notwendige und hinreichende Bedingungen für geringe relative Nachteile Älterer in der Weiterbildung (WBREL) untersucht.

Eine beschäftigungsfördernde Arbeitsmarktpolitik ist keine notwendige Bedingung für geringe Weiterbildungsnachteile Älterer, weder in Ländern mit hohem noch in jenen mit niedrigem Weiterbildungsniveau. Demnach können - entgegen der Erwartung (H1) - die Nachteile Älterer in der Weiterbildung auch in Ländern gering sein, in denen die Arbeitsmarktpolitik wenig beschäftigungsfördernd ist. ${ }^{10}$

Um die Kombinationen von Bedingungen aufzudecken, die zu WBREL führen, wird für jede empirisch beobachtbare Konfiguration (Tab. 5 im Anhang) entschieden, ob diese hinreichend für WBREL ist. ${ }^{11}$ Drei Konfigurationen werden als hinreichend für das Outcome identifiziert (Tab. 3): Die erste Konfiguration qual ${ }^{12} *$ WBSTR beschreibt eine enge Verbindung von Qualifikation und Beschäftigung, also ein duales Ausbildungssystem, mit gut ausgebauten staatlichen und betrieblichen Weiterbildungsstrukturen. In der zweiten Konfiguration AMP *WBSTR ist eine beschäftigungsfördernde Arbeitsmarktpolitik mit guten Weiterbildungsstrukturen kombiniert, die zu geringen relativen Weiterbildungsnachteilen Älterer führt. Die dritte Konfiguration amp * qual * inkl enthält eine wenig beschäftigungsfördernde Arbeitsmarktpolitik, verbunden mit einer engen Kopplung von Qualifikation und Beschäftigung sowie wenig inkludierenden staatlichen Regulierungen bezüglich Älterer. Die Konsistenz ist mit 0,867 insgesamt sehr hoch und deutet darauf hin, dass die Bedingung zu einem hohen Grad hinreichend für WBREL ist. Die Gesamtabde-

\footnotetext{
10 Die Konsistenzwerte aller Bedingungen liegen unter dem benötigten Schwellenwert für notwendige Bedingungen von 0,9 (Schneider und Wagemann 2007, S. 213) und zeigen damit, dass das Outcome keine Teilmenge der Bedingungen ist.

11 Für die Analyse der hinreichenden Bedingungen sollten die Konsistenzwerte der Konfigurationen mindestens 0,75 betragen (Schneider und Wagemann 2007).

12 Die Abwesenheit einer Bedingung wird klein geschrieben.
} 


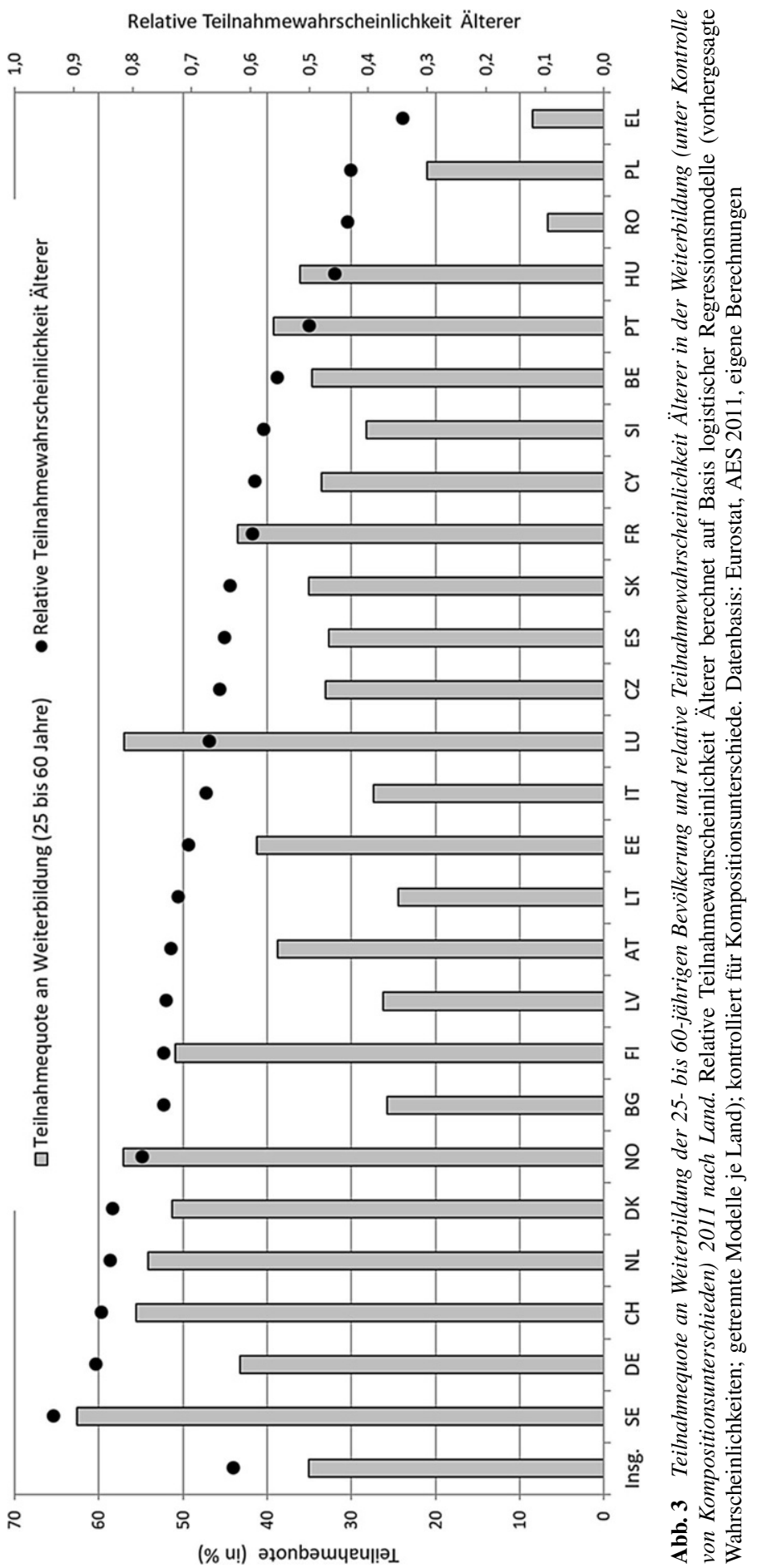


Tab. 3 Hinreichende Bedingungen für geringe relative Nachteile Älterer in der Weiterbildung (WBREL) in Ländern mit hohem Weiterbildungsniveau

\begin{tabular}{|c|c|c|c|c|}
\hline Lösungspfad & Konsistenz & Abdeckung & $\begin{array}{l}\text { Alleinige } \\
\text { Abdeckung }\end{array}$ & Länder \\
\hline (1) qual*WBSTR & 0,956 & 0,547 & 0,086 & $\begin{array}{l}\text { Niederlande, Luxemburg, Norwegen, } \\
\text { Deutschland, Dänemark, Österreich }\end{array}$ \\
\hline $\begin{array}{l}\text { (2) AMP* } \\
\text { WBSTR }\end{array}$ & 0,887 & 0,492 & 0,099 & $\begin{array}{l}\text { Niederlande, Deutschland, Finnland, } \\
\text { Schweden, Frankreich }\end{array}$ \\
\hline (3) amp*qual*inkl & 0,874 & 0,376 & 0,060 & Luxemburg, Norwegen, Schweiz, Ungarn \\
\hline
\end{tabular}

Nicht konsistente Fälle sind kursiv gesetzt; Ergebnisse auf Basis der „Complex Solution“. Datenbasis: Eurostat, AES 2011, eigene Berechnungen

ckung von 0,706 zeigt, dass alle hinreichenden Bedingungen zusammen etwa $71 \%$ des Outcomes erklären.

Die ersten beiden Konfigurationen haben gemeinsam, dass sie beide eine gute staatliche und betriebliche Weiterbildungsstruktur enthalten, während diese in der ersten mit einer engen Verbindung zwischen Qualifikation und Arbeitsmarkt, in der zweiten mit einer beschäftigungsfördernden Arbeitsmarktpolitik kombiniert ist. In diesen Ländern bewirken also gute Weiterbildungsstrukturen in Verbindung mit Bildungsinstitutionen und/oder Arbeitsmarktinstitutionen geringe Nachteile Älterer in der Weiterbildung. Die dritte Konfiguration besitzt mit einer Abdeckung von 0,376 die geringste Erklärungskraft, während die erste die größte Erklärungskraft $(0,547)$ aller aufweist. Deutschland, Luxemburg, die Niederlande und Norwegen sind in mehreren Konfigurationen enthalten. In diesen Ländern existieren also funktionale Äquivalente der hier untersuchten Institutionen und damit verschiedene Möglichkeiten, um geringe Weiterbildungsnachteile Älterer zu erreichen. ${ }^{13}$

Abb. 4 zeigt die Mitgliedschaftswerte der Länder in WBREL und in der gesamten Lösung. Die Mehrheit der Fälle ist konsistent und befindet sich oberhalb der Diagonalen im rechten oberen Quadranten, was die hohe Gesamtkonsistenz bestätigt. Es lassen sich 11 von 13 Fällen erklären, lediglich für Estland und Portugal finden sich keine hinreichenden Bedingungen. In diesen beiden Ländern spielen offenbar andere Faktoren für die Ausprägung der Weiterbildungsnachteile Älterer eine Rolle als die hier untersuchten. Frankreich und Ungarn widersprechen zudem der hinreichenden Bedingung, da diese Länder zwar Mitglied in der Konfiguration sind, gleichzeitig aber eine zu geringe Mitgliedschaft im Outcome aufweisen.

Für die Länder mit niedrigem Weiterbildungsniveau ist dagegen keine der hier untersuchten Bedingungen hinreichend für WBREL, da keine Konfiguration eine ausreichende Konsistenz aufweist (Tab. 6 im Anhang). Dass sich geringe Weiterbildungsnachteile Älterer in Ländern, in denen das Weiterbildungsniveau niedrig ist, nicht durch die in die Analyse einbezogenen Institutionen erklären lassen, verweist auf zusätzlichen Forschungsbedarf und den nötigen Einbezug weiterer hier nicht erfasster Faktoren.

Die Ergebnisse bestätigen, dass staatliche und betriebliche Unterstützung von Weiterbildung - in Kombination mit weiteren Institutionen - eine herausragende

13 Daher ist die alleinige Abdeckung der einzelnen Konfigurationen vergleichsweise gering. 


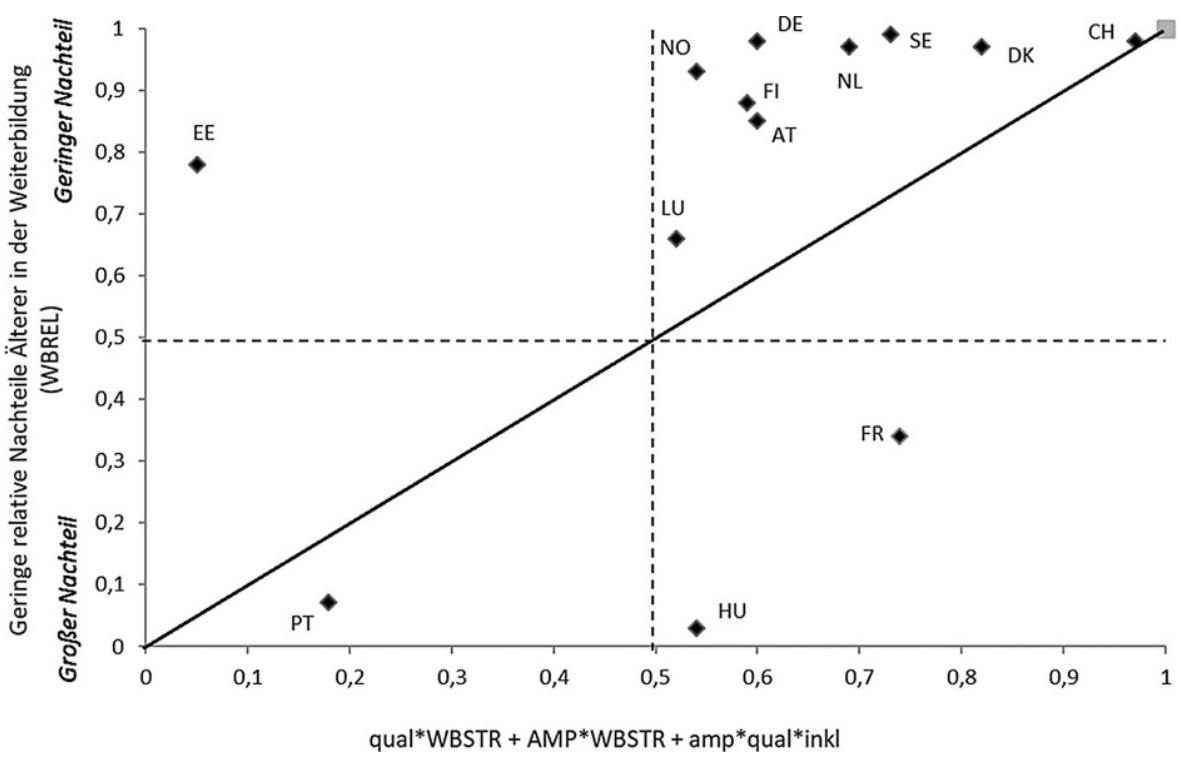

Abb. 4 Hinreichende Bedingungen für geringe relative Nachteile Älterer in der Weiterbildung (WBREL) in Ländern mit hohem Weiterbildungsniveau. Fälle oberhalb und auf der Diagonale sind konsistent. Datenbasis: Eurostat, AES 2011, eigene Berechnungen

Bedeutung für geringe relative Weiterbildungsnachteile Älterer hat, da sich dies in Ländern mit hohem Weiterbildungsniveau als hinreichende Bedingung für WBREL zeigt (H3). Auch eine beschäftigungsfördernde Arbeitsmarktpolitik führt zu geringen Weiterbildungsnachteilen Älterer gegenüber Jüngeren, sie ist aber keine notwendige Bedingung und somit keine Voraussetzung dafür (H1).

Entgegen der Annahme finden sich keine Hinweise darauf, dass eine lockere Verbindung zwischen Qualifikation und Beschäftigungssystem entscheidend für geringe Nachteile Älterer ist, um Defizite aus der Erstausbildung durch Weiterbildung auszugleichen (H2). Stattdessen ist eine enge Verzahnung im Zusammenhang mit weiteren Faktoren für geringe Weiterbildungsnachteile Älterer verantwortlich. Dies kann damit zusammenhängen, dass eine lockere Verbindung zwar für Jüngere mehr Weiterbildung hervorruft, für Ältere aufgrund der lange zurückliegenden Erstausbildung aber nicht; daher gibt es einen größeren Nachteil für Ältere. Zudem kann in den Ländern mit eng gekoppelter Qualifikation und Beschäftigung eine starke Betriebsbindung bei gleichzeitig hohem Bedarf bestehen, ältere Beschäftigte im Betrieb zu halten und diese weiterzubilden.

Inkludierende staatliche Regulierungen scheinen dagegen keine besondere Rolle für die Erklärung geringer relativer Nachteile Älterer zu spielen, da sich diese in keiner der gefundenen Konfigurationen finden (H4). So können exkludierende Regulierungen bezüglich Älterer (kombiniert mit $a m p$ und qual) in manchen Ländern zu WBREL führen, jedoch ist die Erklärungskraft dieser Konfiguration gegenüber den anderen beiden gering und bleibt erklärungsbedürftig. Dies kann an der Definition des hier verwendeten Faktorenbündels liegen, das aufgrund der begrenzten 
Informationen zu Wirkung und Umfang der hier aufgenommenen Maßnahmen für Ältere einen recht engen Ausschnitt inkludierender Regulierungen darstellt.

Darüber hinaus treten alle untersuchten Bedingungen niemals als Einzelfaktoren als hinreichend für das Outcome auf, sondern immer in Kombination mit weiteren Faktoren. Dies entspricht der Annahme (H5) und unterstreicht, dass es kombinierte Effekte von institutionellen Konfigurationen auf relative Weiterbildungsnachteile Älterer gibt.

\section{Diskussion}

Mit dieser Untersuchung ließen sich Zusammenhänge aufdecken, die die bedeutende Rolle institutioneller Konstellationen des Arbeitsmarkts und des Bildungssystems für die Weiterbildungsteilnahme Älterer unterstreichen. Gleichzeitig ist darauf zu verweisen, dass sich die Befunde nur auf die hier untersuchten institutionellen Bedingungen beziehen, für die geeignete Daten verfügbar waren. Ebenso lassen sich die Ergebnisse nur begrenzt auf andere Länder übertragen.

Für einige der betrachteten Länder zeigt sich, dass es nicht die direkte Unterstützung speziell für Ältere, sondern die vorhandenen staatlichen und betrieblichen Weiterbildungsstrukturen sind, die auch die Weiterbildung der Älteren im Vergleich zu Jüngeren fördern. Dies ist in allen skandinavischen Ländern der Fall, ebenso in Deutschland, Luxemburg, den Niederlanden und Österreich. In den Lösungen finden sich viele der konservativen Wohlfahrtsstaaten und alle skandinavischen Länder sowie die Niederlande wieder. Anders als die Mehrheit der süd- und osteuropäischen Länder ist in diesen Ländern die Erklärungskraft gut. Dies verweist auf Ähnlichkeiten zu Wohlfahrtsstaatstypen (Esping-Andersen 1990) und Varieties of capitalism bzw. skill formation systems (z. B. Hall und Soskice 2001; Estevez-Abe et al. 2001). Gleichzeitig trennen sich die Ergebnisse für konservative Wohlfahrtsstaaten in jene mit dualem Ausbildungssystem (Deutschland und Österreich), die gut durch die Analyse erklärbar sind, und jene ohne dieses (Belgien und Frankreich). Trotz der Ähnlichkeiten zu bekannten Typologien lassen sich die Nachteile Älterer in der Weiterbildung also nicht allein durch diese deuten.

Um Ursachen für Nachteile in der Weiterbildungsbeteiligung zu erforschen, müssen zukünftig weitere Institutionen des Arbeitsmarktes und des Bildungssystems sowie differenzielle Erklärungsansätze bezüglich des Weiterbildungsniveaus berücksichtigt werden. Auch ergeben sich Hinweise zu den teilweise unklaren Ergebnissen der bisherigen Forschung, die lediglich einzelne Institutionen in den Blick nehmen (Abschn. 2.1). Die mitunter konträren Befunde können gerade durch die Nichtbeachtung des Zusammenspiels mehrerer Institutionen zustande kommen.

Generell spricht die Tatsache, dass Institutionen ausschließlich in Kombination miteinander geringe relative Nachteile Älterer in der Weiterbildung bewirken, dafür, dass sich Maßnahmen zur Förderung der Weiterbildungsbeteiligung Älterer - und vermutlich auch anderer in der Weiterbildung unterrepräsentierter Bevölkerungsgruppen - immer auf die institutionellen Konfigurationen im Land beziehen sollten. Ebenfalls zeigen sich in manchen Ländern alternative Handlungsmöglichkeiten hinsichtlich sozialpolitischer Maßnahmen, um die Nachteile Älterer in der 
Weiterbildung zu verringern. Dabei sollten insbesondere Weiterbildungsstrukturen auf staatlicher und betrieblicher Ebene insgesamt verbessert werden, denn die für alle zugänglichen Weiterbildungsangebote sind nach der vorliegenden Studie entscheidend für das Weiterbildungsverhalten von Personen in der späten Erwerbsphase. Somit sind nicht nur Maßnahmen wie Weiterbildungsprogramme speziell für Ältere zum Abbau von Disparitäten Älterer in der Weiterbildung wichtig, sondern der Ausbau allgemeiner öffentlicher und betrieblicher Weiterbildungsangebote. Der Staat kann hier betriebliche Angebote und Maßnahmen fördern, um vor allem Anreize für die bisher in der Weiterbildung unterrepräsentierten kleinen und mittleren Betriebe zu schaffen.

Open Access Dieser Artikel wird unter der Creative Commons Namensnennung 4.0 International Lizenz veröffentlicht, welche die Nutzung, Vervielfältigung, Bearbeitung, Verbreitung und Wiedergabe in jeglichem Medium und Format erlaubt, sofern Sie den/die ursprünglichen Autor(en) und die Quelle ordnungsgemäß nennen, einen Link zur Creative Commons Lizenz beifügen und angeben, ob Änderungen vorgenommen wurden.

Die in diesem Artikel enthaltenen Bilder und sonstiges Drittmaterial unterliegen ebenfalls der genannten Creative Commons Lizenz, sofern sich aus der Abbildungslegende nichts anderes ergibt. Sofern das betreffende Material nicht unter der genannten Creative Commons Lizenz steht und die betreffende Handlung nicht nach gesetzlichen Vorschriften erlaubt ist, ist für die oben aufgeführten Weiterverwendungen des Materials die Einwilligung des jeweiligen Rechteinhabers einzuholen.

Weitere Details zur Lizenz entnehmen Sie bitte der Lizenzinformation auf http://creativecommons.org/ licenses/by/4.0/deed.de. 


\section{Anhang}

Tab. 4 Fuzzy-Werte für Outcome und institutionelle Bedingungen

\begin{tabular}{|c|c|c|c|c|c|c|c|c|c|}
\hline Land & $\begin{array}{l}\text { Outcome } \\
W B R E L\end{array}$ & $A K T$ & BESCH & $\begin{array}{l}\mathrm{FB}^{\mathrm{a}} \\
A M P\end{array}$ & $\begin{array}{l}\mathrm{FB}^{\mathrm{a}} \\
Q U A L\end{array}$ & \multicolumn{3}{|c|}{ WBSTAAT WBBETR $\mathrm{FB}^{\mathrm{a}}$} & \multirow[t]{2}{*}{$\begin{array}{l}\mathrm{FB}^{\mathrm{a}} \\
I N K L\end{array}$} \\
\hline \multicolumn{9}{|c|}{ Länder mit hohem Weiterbildungsniveau } & \\
\hline DK & 0,97 & 0,98 & 0,24 & 0,24 & 0,03 & 1,0 & 0,82 & 0,82 & 0,7 \\
\hline $\mathrm{DE}$ & 0,98 & 0,75 & 0,75 & 0,75 & 0,03 & 0,6 & 0,84 & 0,60 & 1,0 \\
\hline $\mathrm{EE}$ & 0,78 & 0,01 & 0,08 & 0,01 & 0,95 & 0,4 & 0,09 & 0,09 & 0 \\
\hline FI & 0,88 & 0,68 & 0,59 & 0,59 & 0,53 & 1,0 & 0,60 & 0,60 & 0,3 \\
\hline FR & 0,34 & 0,74 & 0,81 & 0,74 & 0,59 & 0,8 & 0,96 & 0,80 & 0,7 \\
\hline LU & 0,66 & 0,66 & 0,47 & 0,47 & 0,48 & 0,8 & 0,90 & 0,80 & 0,3 \\
\hline NL & 0,97 & 0,98 & 0,93 & 0,93 & 0,31 & 0,8 & 0,98 & 0,80 & 0,3 \\
\hline $\mathrm{NO}$ & 0,93 & 0,90 & 0,42 & 0,42 & 0,46 & 1,0 & 0,67 & 0,67 & 0 \\
\hline AT & 0,85 & 0,89 & 0,31 & 0,31 & 0,08 & 0,6 & 0,94 & 0,60 & 0,7 \\
\hline PT & 0,07 & 0,18 & 1,00 & 0,18 & 0,95 & 0,4 & 0,42 & 0,40 & 0,7 \\
\hline SE & 0,99 & 0,73 & 0,74 & 0,73 & 0,95 & 1,0 & 0,96 & 0,96 & 0 \\
\hline $\mathrm{CH}$ & 0,98 & 0,78 & 0,03 & 0,03 & 0 & 0,4 & 0,85 & 0,40 & 0 \\
\hline $\mathrm{HU}$ & 0,03 & 0,17 & 0,11 & 0,11 & 0,46 & 0,2 & 0,22 & 0,20 & 0,3 \\
\hline \multicolumn{10}{|c|}{ Länder mit niedrigem Weiterbildungsniveau } \\
\hline $\mathrm{BE}$ & 0,19 & 0,56 & 0,53 & 0,53 & 0,91 & 0,6 & 0,97 & 0,60 & 1,0 \\
\hline BG & 0,88 & 0,01 & 0,11 & 0,01 & 0,95 & 0,2 & 0,04 & 0,04 & 0,7 \\
\hline EL & 0 & 0,02 & 0,87 & 0,02 & 0,95 & 0,2 & 0,06 & 0,06 & 0,3 \\
\hline IT & 0,68 & 0,07 & 0,81 & 0,07 & 0,95 & 0,4 & 0,15 & 0,15 & 0,7 \\
\hline LV & 0,87 & 0,03 & 0,78 & 0,03 & 0,95 & 0,2 & 0,04 & 0,04 & 0,3 \\
\hline LT & 0,82 & 0,02 & 0,42 & 0,02 & 0,95 & 0,4 & 0,06 & 0,06 & 0,3 \\
\hline PL & 0,02 & 0,34 & 0,39 & 0,34 & 0,83 & 0,2 & 0,10 & 0,10 & 0,3 \\
\hline RO & 0,02 & 0,01 & 0,38 & 0,01 & 0,94 & 0,2 & 0,02 & 0,02 & 0,7 \\
\hline SK & 0,52 & 0,02 & 0,38 & 0,02 & 0,15 & 0 & 0,33 & 0 & 0,3 \\
\hline SI & 0,27 & 0,31 & 0,65 & 0,31 & 0,95 & 0,2 & 0,59 & 0,20 & 0,3 \\
\hline ES & 0,56 & 0,08 & 0,41 & 0,08 & 0,95 & 0,4 & 0,28 & 0,28 & 0,7 \\
\hline $\mathrm{CZ}$ & 0,59 & 0,08 & 0,97 & 0,08 & 0,11 & 0,2 & 0,16 & 0,16 & 0 \\
\hline CY & 0,32 & 0,09 & 0,07 & 0,07 & 0,95 & 0,6 & 0,34 & 0,34 & 0,3 \\
\hline
\end{tabular}

${ }^{\mathrm{a}} F B$ Faktorenbündel

Datenbasis: Eurostat, AES 2011, eigene Berechnungen 
Tab. 5 Wahrheitstabelle für geringe relative Nachteile Älterer in der Weiterbildung (WBREL) in Ländern mit hohem Weiterbildungsniveau

\begin{tabular}{llllllll}
\hline AMP & QUAL & WBSTR & INKL & WBREL & Fallzahl & Konsistenz & Länder \\
\hline 1 & 0 & 1 & 0 & 1 & 1 & 0,975 & Niederlande \\
0 & 0 & 1 & 0 & 1 & 2 & 0,946 & $\begin{array}{l}\text { Luxemburg, Norwe- } \\
\text { gen }\end{array}$ \\
1 & 0 & 1 & 1 & 1 & 1 & 0,943 & Deutschland \\
1 & 1 & 1 & 0 & 1 & 2 & 0,940 & Finnland, Schweden \\
0 & 0 & 1 & 1 & 1 & 2 & 0,938 & Dänemark, Öster- \\
& & & & & & & reich \\
0 & 0 & 0 & 0 & 1 & 2 & 0,828 & Schweiz, Ungarn \\
1 & 1 & 1 & 1 & 1 & 1 & 0,771 & Frankreich \\
0 & 1 & 0 & 0 & 0 & 1 & 0,738 & Estland \\
0 & 1 & 0 & 1 & 0 & 1 & 0,558 & Portugal \\
\hline
\end{tabular}

Anmerkung: Die Werte 1 und 0 geben an, ob die jeweiligen Bedingungen vorhanden (Wert 1) oder nicht vorhanden (Wert 0) sind. Jede Zeile zeigt eine institutionelle Konfiguration, die im Sample vorhanden ist; fehlende Zeilen weisen auf Konfigurationen ohne empirische Fälle hin. Datenbasis: Eurostat, AES 2011, eigene Berechnungen

Tab. 6 Wahrheitstabelle für geringe relative Nachteile Älterer in der Weiterbildung (WBREL) in Ländern mit niedrigem Weiterbildungsniveau

\begin{tabular}{llllllll}
\hline AMP & QUAL & WBSTR & INKL & WBREL & Fallzahl & Konsistenz & Länder \\
\hline 0 & 0 & 0 & 0 & 0 & 2 & 0,694 & $\begin{array}{l}\text { Slowakei, Tschechien } \\
\text { Bulgarien, Italien, Rumäni- } \\
\text { en, Spanien }\end{array}$ \\
1 & 1 & 0 & 1 & 0 & 4 & 0,678 & Belgien \\
0 & 1 & 1 & 1 & 0 & 1 & 0,614 & $\begin{array}{l}\text { Griechenland, Lettland, } \\
\text { Litauen, Polen, Slowenien, } \\
\text { Zypern }\end{array}$ \\
\hline
\end{tabular}

Anmerkung: Die Werte 1 und 0 geben an, ob die jeweiligen Bedingungen vorhanden (Wert 1) oder nicht vorhanden (Wert 0) sind. Jede Zeile zeigt eine institutionelle Konfiguration, die im Sample vorhanden ist; fehlende Zeilen weisen auf Konfigurationen ohne empirische Fälle hin. Datenbasis: Eurostat, AES 2011, eigene Berechnungen 


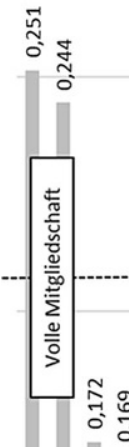

o.

ำ

ษ 귄

今

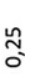

吾

=

究

₹:

春

ฐี

ํํำ

รัำ

宊

离

守

这

ํㅗㅇ

$\stackrel{ }{\Xi}$.
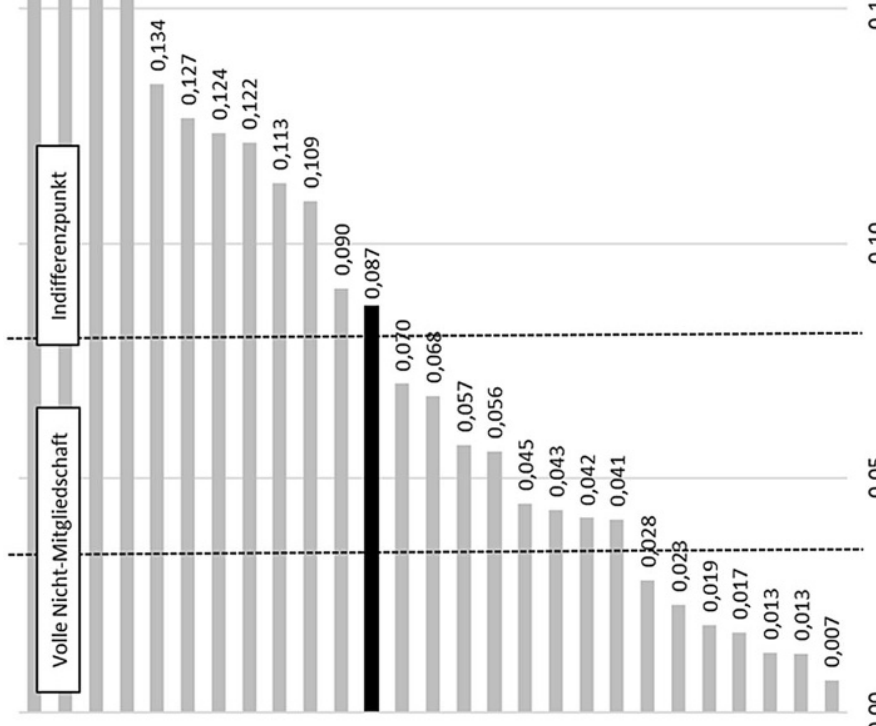

옹

จิ

¿

웡

ฐั

跤

s

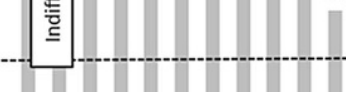

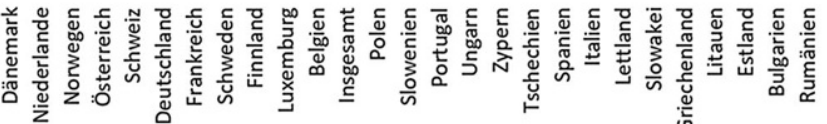

ะั

:

竞

ก

: ะี

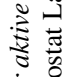

空害品

8

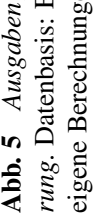




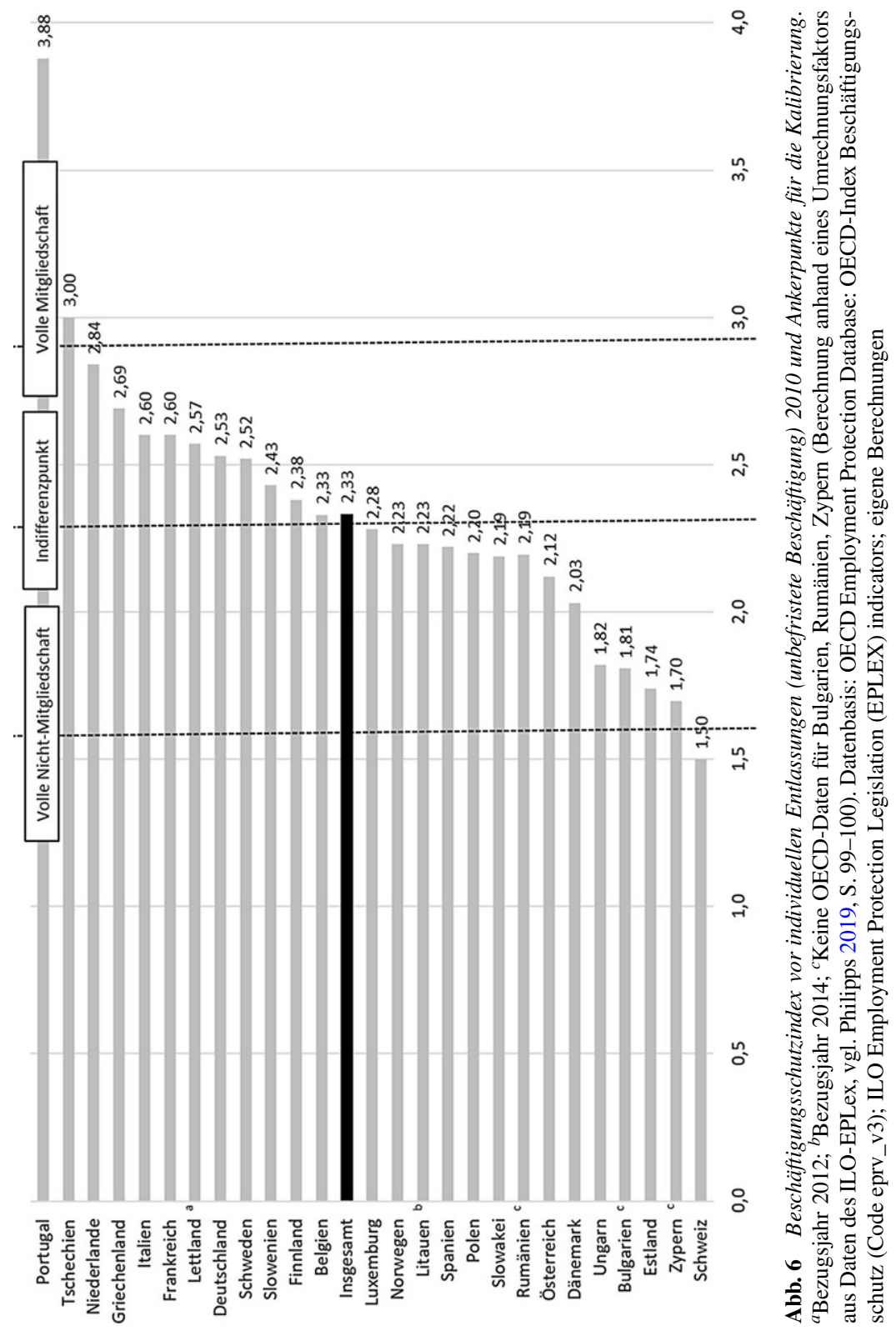




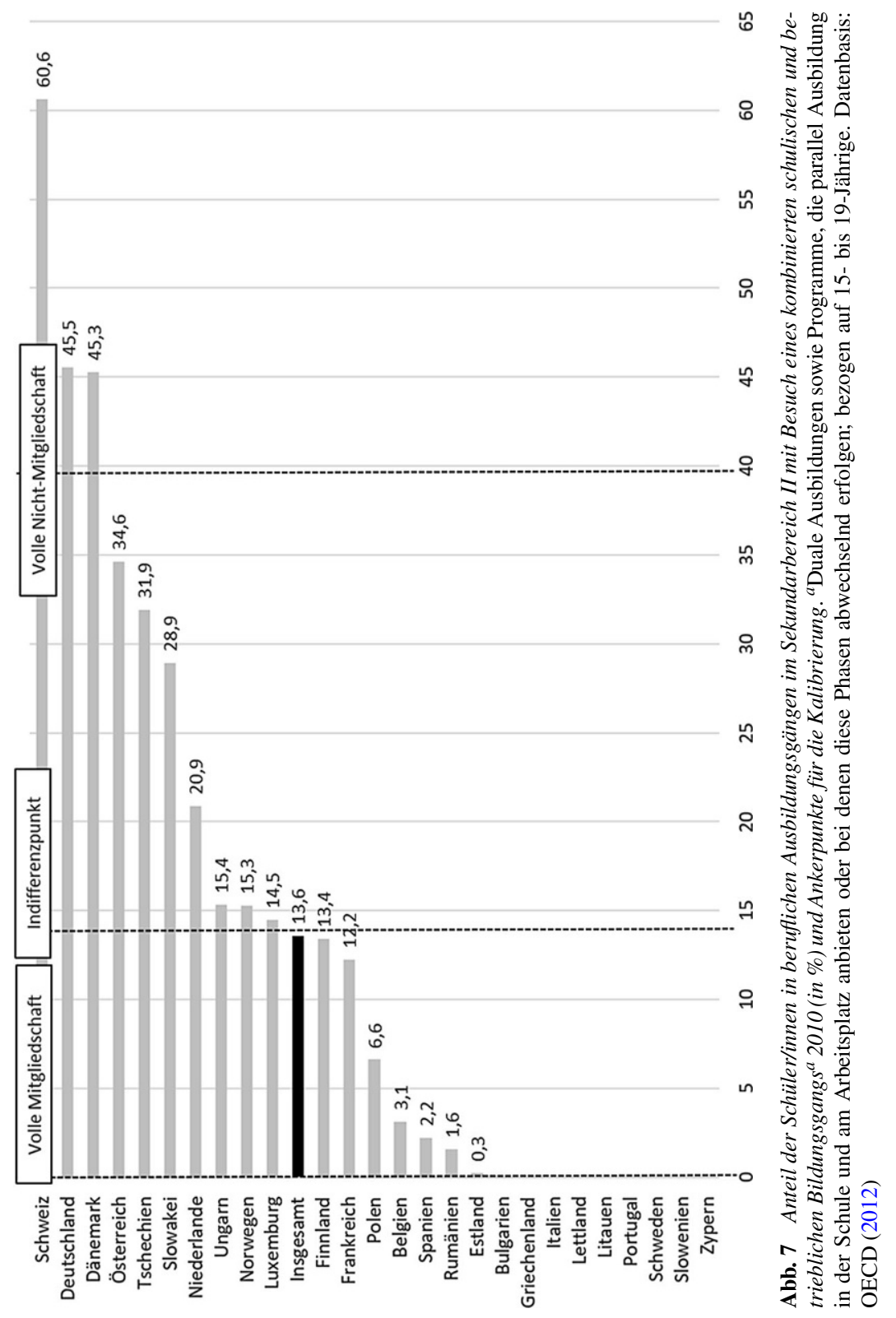




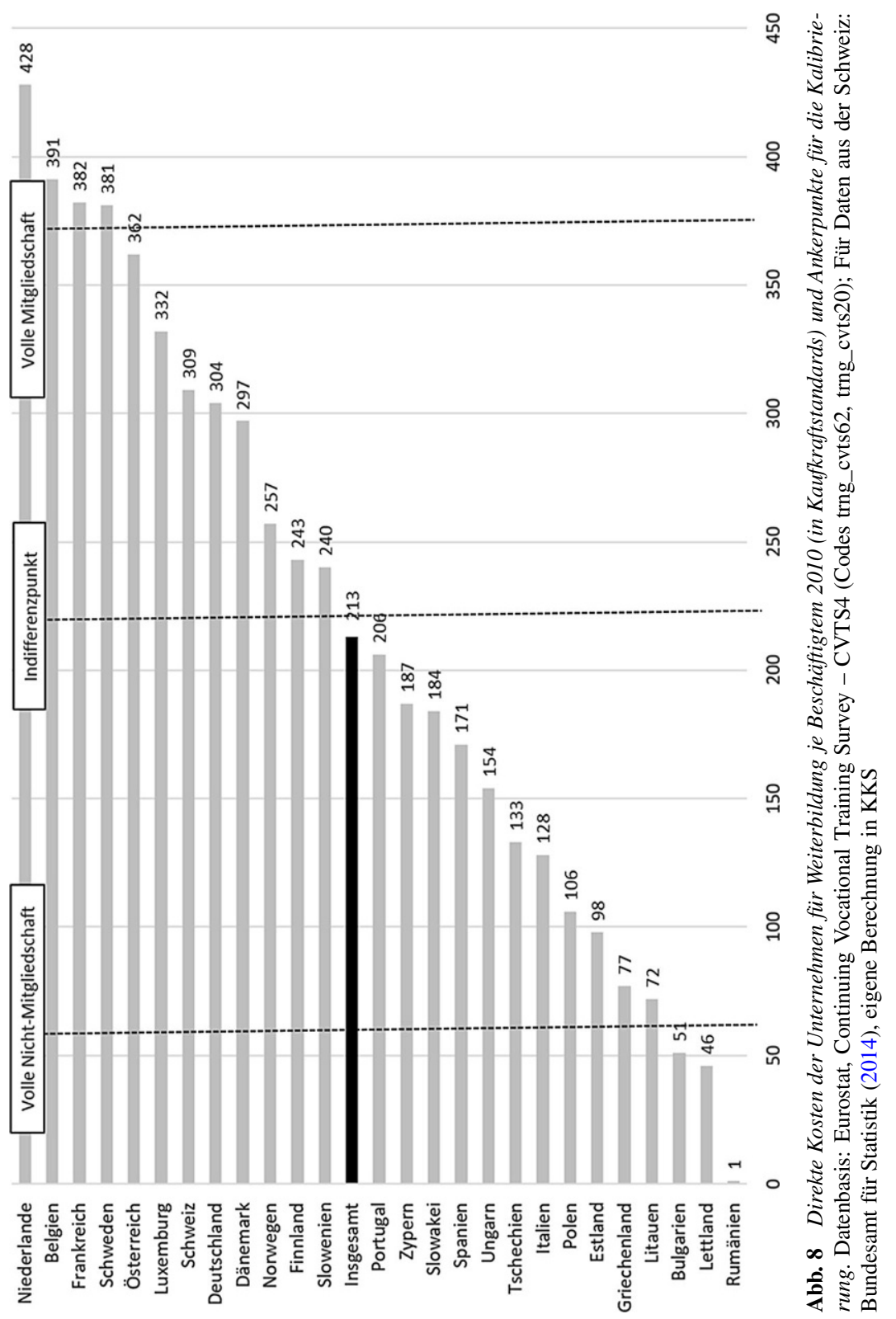




\section{Literatur}

Acemoglu, D., \& Pischke, J.-S. (1999). Beyond Becker. Training in imperfect labour markets. The Economic Journal, 109, 112-142.

Allmendinger, J. (1989). Educational systems and labor market outcomes. European Sociological Review, 5(3), 231-250.

Almeida, R. K., \& Aterido, R. (2008). The incentives to invest in job training: do strict labor codes influence this decision? Social protection discussion paper, Bd. 0832. Washington, DC: World Bank.

Arulampalam, W., Booth, A.L., \& Bryan, M.L. (2004). Training in Europe. Journal of the European Economic Association, 2(2/3), 346-360.

Bassanini, A. (2006). Training, wages and employment security: an empirical analysis on European data. Applied Economics Letters, 13, 523-527.

Bassanini, A., Booth, A., Brunello, G., De Paola, M., \& Leuven, E. (2005). Workplace training in Europe. IZA discussion paper, Bd. 1640. Bonn: Forschungsinstitut zur Zukunft der Arbeit.

Becker, G. S. (1964). Human capital. A theoretical and empirical analysis with special reference to education. New York: NBER.

Becker, R. (2018). Berufliche Weiterbildung im Arbeitsmarkt. In M. Abraham \& T. Hinz (Hrsg.), Arbeitsmarktsoziologie (S. 311-353). Wiesbaden: Springer VS.

Behringer, F., \& Schönfeld, G. (2014). Schwerpunktthema: Lernen Erwachsener in Deutschland im europäischen Vergleich. In Bundesinstitut für Berufsbildung (Hrsg.), Datenreport zum Berufsbildungsbericht 2014. Informationen und Analysen zur Entwicklung der beruflichen Bildung (S. 381-413). Bielefeld: W. Bertelsmann.

Boeren, E. (2016). Lifelong learning participation in a changing policy context: an interdisciplinary theory. Basingstoke: Palgrave Macmillan.

Boeren, E., \& Holford, J. (2016). Vocationalism varies (a lot): a 12-country multivariate analysis of participation in formal adult learning. Adult Education Quarterly, 66(2), 120-142.

Borgna, C. (2017). Different systems, same inequalities? Post-compulsory education and young adults' literacy in 18 OECD-countries. Journal of European Social Policy, 27(4), 332-345.

Brunello, G. (2004). Labour market institutions and the complementarity between education and training in Europe. In D. Checchi \& C. Lucifora (Hrsg.), Education, training and labour market outcomes in Europe (S. 188-210). London: Palgrave Macmillian.

Buche, J., \& Siewert, M. B. (2015). Qualitative Comparative Analysis (QCA) in der Soziologie. Perspektiven, Potentiale und Anwendungsbereiche. Zeitschrift für Soziologie, 44(6), 386-406.

Bundesamt für Statistik (2014). Berufliche Weiterbildung in Unternehmen der Schweiz. Ergebnisse der Erhebung zur beruflichen Weiterbildung in Unternehmen 2011 (S. 23). Neuchâtel: Bundesamt für Statistik.

Coulombe, S., \& Tremblay, J.-F. (2007). Explaining cross-country differences in job-related training: Macroeconomic evidence from OECD countries. Economie internationale, 110(2), 5-29.

Cross, P. K. (1981). Adults as learners. Increasing participation and facilitating learning. San Francisco, London: Jossey-Bass.

Culpepper, P.D., \& Thelen, K. (2008). Institutions and collective actors in the provision of training. Historical and cross-national comparisons. In K. U. Mayer \& H. Solga (Hrsg.), Skill Formation. Interdisciplinary and cross-national perspectives (S. 21-49). New York: Cambridge University Press.

Dämmrich, J., Vono de Vilhena, D., \& Reichart, E. (2014). Participation in adult learning in Europe: the impact of country-level and individual characteristics. In H.-P. Blossfeld (Hrsg.), Adult learning in modern societies. An international comparison from a life-course perspective (S. 29-55). Cheltenham: Edward Elgar.

Dieckhoff, M. (2007). Does it work? The effect of continuing training on labour market outcomes: a comparative study of Germany, Denmark and the United Kingdom. European Sociological Review, 23(3), 295-308.

Dieckhoff, M., \& Steiber, N. (2011). A re-assessment of common theoretical approaches to explain gender differences in continuing training participation. British Journal of Industrial Relations, 49, 135-157.

Dieckhoff, M., Jungblut, J.-M., \& O’Connell, P. J. (2007). Job-related training in Europe: Do institutions matter? In D. Gallie (Hrsg.), Employment regimes and the quality of work (S. 77-103). Oxford: Oxford University Press.

Dietz, M., \& Walwei, U. (2011). Germany. No country for old workers? Zeitschrift für Arbeitsmarktforschung, 44, 363-376. 
Ebbinghaus, B., \& Hofäcker, D. (2013). Trendwende bei der Frühverrentung in modernen Wohlfahrtsstaaten. Paradigmenwechsel zur Überwindung von Push- und Pull-Faktoren. Comparative Population Studies - Zeitschrift für Bevölkerungswissenschaft, 38(4), 841-880.

Esping-Andersen, G. (1990). The three worlds of welfare capitalism. Cambridge: Polity Press.

Estevez-Abe, M., Iversen, T., \& Soskice, D. (2001). Social protection and the formation of skills: a reinterpretation of the welfare state. In P. A. Hall \& D. Soskice (Hrsg.), Varieties of capitalism. The institutional foundations of comparative advantage (S. 145-183). Oxford: OUP.

Eurofound (2013). Role of governments and social partners in keeping older workers in the labour market. Dublin: European Foundation for the Improvement of Living and Working Conditions.

European Commission, \& Eurostat (2013). Draft Adult Education Survey Manual. Version 9. https:// circabc.europa.eu/sd/a/23580311-3517-422b-9588-b365c10dc440/AES_MANUAL_2013_November. pdf. Zugegriffen: 27. Jan. 2020.

Evans, K., Schoon, I., \& Weale, M. (2012). Life chances, learning and the dynamics of risk in the life course. In D. N. Aspin (Hrsg.), Second international handbook of lifelong learning (S. 245-268). Dordrecht: Springer.

Fourage, D., \& Schils, T. (2009). The effect of early retirement incentives on the training participation of older workers. Labour, 23(Special Issue), 85-109.

Groenez, S., Desmedt, E., \& Nicaise, I. (2007). Participation in lifelong learning in the EU-15: the role of macro-level determinants. http://www.academia.edu/333827/Participation_In_Lifelong_Learning_ In_the_EU-15_The_Role_of_Macro-Level_Determinants. Zugegriffen: 27. Jan. 2020. Paper for the ECER Conference, Ghent, Belgium, September 2007.

Hall, P. A., \& Soskice, D. (2001). An introduction to varieties of capitalism. In P. A. Hall \& D. Soskice (Hrsg.), Varieties of capitalism. The institutional foundations of comparative advantage (S. 1-68). Oxford: OUP.

Heinz, W. R. (1992). Introduction: institutions and gatekeeping in the life course. In W. R. Heinz (Hrsg.), Institutions and gatekeeping in the life course (S. 9-27). Weinheim: Deutscher Studien Verlag.

Ioannidou, A., \& Knauber, C. (2019). Adult literacy and basic education policies in a comparative perspective. Selected findings from four country cases. Studies in Adult Education and Learning, 25(3), 125-140.

Kaufmann, K., \& Widany, S. (2013). Berufliche Weiterbildung. Gelegenheits- und Teilnahmestrukturen. Zeitschrift für Erziehungswissenschaft, 16, 29-54.

Knauber, C. (2017). International-vergleichende Forschung zur Bildungspolitik: Literatur-Review theoretischer Ansätze und Perspektiven der vergleichenden Politikwissenschaft. Zeitschrift für Weiterbildungsforschung, 40, 171-198.

Knipprath, H., \& De Rick, K. (2015). How social and human capital predict participation in lifelong learning: a longitudinal data analysis. Adult Education Quarterly, 65(1), 50-66.

Kuper, H., Unger, K., \& Hartmann, J. (2013). Multivariate Analyse zur Weiterbildungsbeteiligung. In F. Bilger (Hrsg.), Weiterbildungsverhalten in Deutschland. Resultate des Adult Education Survey 2012. Theorie und Praxis der Erwachsenenbildung (S. 95-107). Bielefeld: W. Bertelsmann.

Markowitsch, J., \& Hefler, G. (2007). To Train or Not to Train-Explaining differences in average enterprise training performance in Europe. A framework approach. http://trainingineurope.com/mmedia/ 2007.10.22/1193059438.pdf. Zugegriffen: 27. Jan. 2020.

Martin, A., \& Rüber, I.E. (2016). Die Weiterbildungsbeteiligung von Geringqualifizierten im internationalen Vergleich - Eine Mehrebenenanalyse. Zeitschrift für Weiterbildungsforschung - Report, 39, 149-169.

Mayer, K. U., \& Solga, H. (2008). Skill Formation. Interdisciplinary and crossnational perspectives. In K. U. Mayer \& H. Solga (Hrsg.), Skill Formation. Interdisciplinary and cross-national perspectives (S. 1-18). New York: Cambridge University Press.

Moraal, D., \& Schönfeld, G. (2007). Paradigmenwechsel: Weiterbildung statt Frühverrentung? Weiterbildung für ältere Arbeitnehmerinnen, Arbeitnehmer und Arbeitslose im internationalen Vergleich. In P. Kaune, J. Rützel \& G. Spöttl (Hrsg.), Berufliche Bildung, Innovation, soziale Integration: internationale Wettbewerbsfähigkeit, Entwicklung und Karriere, Mitgestaltung von Arbeit und Technik (S. 24-38). Bielefeld: W. Bertelsmann.

Müller, W., \& Shavit, Y. (1998). The institutional embeddedness of the stratification process: a comparative study of qualifications and occupations in thirteen countries. In Y. Shavit \& W. Müller (Hrsg.), From school to work. A comparative study of educational qualifications and occupational destinations (S. 1-48). Oxford: Clarendon. 
O’Connell, P., \& Jungblut, J.-M. (2008). What do we know about training at work? In K. U. Mayer \& H. Solga (Hrsg.), Skill formation. Interdisciplinary and cross-national perspectives (S. 109-125). New York: Cambridge University Press.

OECD (2012). Education at a Glance 2012: OECD indicators (S. 332). Paris: Organisation for Economic Co-operation and Development.

OECD (2013). Statistical annex. In OECD employment outlook 2013 (S. 263). Paris: OECD Publishing.

OECD (2019). Getting skills right: future-ready adult learning systems, getting skills right. Paris: Organisation for Economic Co-operation and Development.

Ok, W., \& Tergeist, P. (2003). Improving workers' skills: analytical evidence and the role of the social partners. OECD social, employment and migration working papers, Bd. 10. Paris: OECD Publishing.

Öztürk, H. (2011). Weiterbildung im Kontext heterogener Lebenswelten. Zeitschrift für Bildungsforschung, $1,151-164$.

Philipps, V. (2019). Die Bedeutung von Institutionen für die Weiterbildung Älterer. Eine vergleichende Studie erwerbsbezogener Weiterbildungsteilnahme in Europa. Wiesbaden: Springer VS.

Ragin, C.C. (1987). The comparative method: moving beyond quantitative and qualitative strategies. Berkeley: University of Berkeley Press.

Ragin, C.C. (2009). Qualitative comparative analysis using fuzzy sets (fsQCA). In B. Rihoux \& C. C. Ragin (Hrsg.), Configurational comparative methods. Qualitative comparative analysis (QCA) and related techniques (S. 87-121). Los Angeles: SAGE.

Ragin, C.C., \& Davey, S. (2016). Fuzzy Set/Qualitative Comparative Analysis 3.0. Irvine: Department of Sociology, University of California

Rubenson, K., \& Desjardins, R. (2009). The impact of welfare state regimes on barriers to participation in adult education: a bounded agency model. Adult Education Quarterly, 59(3), 187-207.

Schneider, C. Q., \& Wagemann, C. (2007). Qualitative Comparative Analysis und Fuzzy Sets. Ein Lehrbuch für Anwender und jene, die es werden wollen. Opladen, Farmington Hills: Barbara Budrich.

Scott, W.R. (2001). Institutions and organizations. Foundations for organizational science. Thousand Oaks: SAGE.

Vogtenhuber, S. (2015). Explaining country variation in employee training: an institutional analysis of education systems and their influence on training and its returns. European Sociological Review, 31(1), 77-90.

Wagemann, C., \& Schneider, C. Q. (2010). Qualitative comparative analysis (QCA) and fuzzy-sets: agenda for a research approach and a data analysis technique. Comparative Sociology, 9, 376-396.

Wolbers, M. H. J. (2005). Initial and further education: substitutes or complements? International Review of Education, 51(5/6), 459-478.

Publisher's Note Springer Nature remains neutral with regard to jurisdictional claims in published maps and institutional affiliations. 